\author{
Leila Lobato Graef
}

\title{
Um Modelo para o Decaimento da Energia Escura
}

Dissertação de mestrado apresentada ao Instituto de Física da Universidade de São Paulo, para a obtenção do título de Mestre em Ciências, sob a orientação do Professor Dr. Élcio Abdalla.

São Paulo

2012 


\section{Agradecimentos}

Agradeço ao meu orientador Prof. Dr. Élcio Abdalla pela oportunidade, pela proposta de pesquisa, pela confiança.

Ao professor Dr. Bin Wang pelos comentários úteis.

Aos meus pais, especialmente, por todo incentivo e apoio que sempre me deram sem o qual este trabalho não haveria sido realizado, e ao meu irmão.

Ao Felipe Carrelli, pela compreensão, pelo incentivo, pela paciência, e por me apoiar em todos os momentos.

À Fátima e ao Álvaro por estarem presentes sempre me ajudando.

Aos professores e monitores que com muita dedicação me acompanharam nesta fase, especialmente ao professor Dr. Emerson Passos e ao Eduardo Matsushita. Também ao professor Dr. Ademir pelas discussões construtivas.

Aos meus grandes amigos Victor Peralta, Daniel Cruz, Leonardo Jerônimo, e todos os outros que estiveram presentes nesta etapa.

Aos amigos Ernandes e Michele pelas dicas importantes.

Ao suporte do Instituto de Física.

$\mathrm{E}$ ao financiamento do CNPQ. 


\section{Resumo}

Neste trabalho discutimos um modelo baseado em teoria de campos para descrever a energia escura, no qual ela é representada por uma partícula ultra-leve situada em um mínimo metaestável de um potencial. Mostramos que a energia escura neste modelo decai em matéria escura durante o tempo de vida do universo, amenizando o problema da coincidência. 


\section{Abstract}

In the present work we discuss a field theory model in which dark energy is described by ultra-light particle situated at a metastable minimum of a potential. We show that dark energy in this model decays into dark matter during a time scale corresponding to the age of the universe, alleviating the coincidence problem. 


\section{Sumário}

1- Introdução 7

1.1- O Modelo Padrão da Cosmologia 7

1.1.1- A Constante Cosmológica 13

2- Quintessência 17

3- A Interação no Setor Escuro do Universo 21

4- Gravitação Modificada 24

5- Um Modelo para a Energia Escura 28

6- O Método Semiclássico do Tunelamento 31

7- O Decaimento da Energia Escura 44

8- Conclusão 56

9- Apêndice $\quad 57$

$\begin{array}{ll}\text { Apêndice A } & 57\end{array}$

Apêndice B $\quad 59$

Apêndice C $\quad 61$

$\begin{array}{ll}\text { Referências Bibliográficas } & 71\end{array}$ 


\section{1- Introdução}

\section{1 - O Modelo Padrão da Cosmologia}

Em 1915 Einstein completou a sua formulação da Teoria da Relatividade Geral, segundo a qual um certo conteúdo de matéria e energia está relacionado a uma propriedade geométrica do espaço-tempo de acordo com a equação

$$
R_{\mu \nu}-\frac{1}{2} g_{\mu \nu} R=8 \pi G T_{\mu \nu} .
$$

O conteúdo de matéria e energia do universo é representado pelo tensor energia-momento $T_{\mu \nu}$. O lado esquerdo da equação representa a geometria gerada pelo conteúdo energético, descrita através da métrica $g_{\mu \nu}$, que é relacionada ao elemento de linha $d s^{2}$ através da expressão

$$
d s^{2}=g_{\mu \nu} d x^{\mu} d x^{\nu},
$$

onde $d s^{2}$ é a distância entre dois eventos no espaço-tempo quadridimensional.

O primeiro termo no lado esquerdo da equação de Einstein é o tensor simétrico de Ricci, $R_{\mu \nu}$, definido em termos dos símbolos de Christoffel da seguinte forma [1]

$$
R_{\mu \nu}=\Gamma_{\mu \beta, \nu}^{\beta}-\Gamma_{\mu \nu, \beta}^{\beta}+\Gamma_{\mu \beta}^{\alpha} \Gamma_{\alpha \nu}^{\beta}-\Gamma_{\mu \nu}^{\alpha} \Gamma_{\alpha \beta}^{\beta} .
$$

Os símbolos de Christoffel acima, por sua vez, são escritos em termos da métrica como [1]

$$
\Gamma_{\mu \beta}^{\sigma}=\frac{g^{\sigma \nu}}{2}\left(g_{\mu \nu, \beta}+g_{\nu \beta, \mu}-g_{\mu \beta, \nu}\right) .
$$


Temos também, no lado esquerdo da equação, o escalar de curvatura $R$ que é dado pela contração

$$
R=R_{\mu}^{\mu}=g^{\mu \alpha} R_{\alpha \mu} .
$$

Com sua teoria já formulada, Einstein buscava uma solução para suas equações que descrevesse um universo estático. Uma das razões para seu interesse em obter uma solução estática para o universo era buscando que suas equações estivessem de acordo com o princípio de Mach, segundo o qual a matéria determina a inércia. Além disso, naquela época não havia dados experimentais que indicassem um universo em evolução, e a crença geral era então a de um universo estático [2].

Porém, não era possível encontrar uma solução descrevendo um universo estático através das equações de Einstein. Um universo preenchido por matéria com pressão nula, como a matéria ordinária que conhecemos, de acordo com estas equações entraria em colapso gravitacional, ou evoluiria aumentando seu tamanho para sempre, não havendo portanto uma solução estática. Einstein então modificou sua equação, incluindo no lado esquerdo o termo da constante cosmológica $\Lambda$,

$$
R_{\mu \nu}-\frac{1}{2} g_{\mu \nu} R+\Lambda g_{\mu \nu}=8 \pi G T_{\mu \nu} .
$$

Desta forma, a equação de Einstein poderia ter soluções estáticas para um universo preenchido por matéria com pressão nula e densidade positiva. Vemos que de fato o lado esquerdo desta equação é a forma mais geral que podemos construir a partir da métrica e sua primeira e segunda derivada, que seja tensorial de ordem 2, local, invariante sob transformações de coordenadas, sem divergência e simétrica.

No entanto, esta equação não era estável, pois qualquer pequeno desvio no balanço dos termos na equação acima rapidamente tornava a solução não estática.

Pouco tempo após a introdução da constante cosmológica, Einstein voltou atrás na sua proposta e retirou a constante cosmológica da sua equação. Com a descoberta do redshift das galáxias, que aumenta com a distância, surgiam evidências de um universo em expansão. Sem a necessidade de se impor uma solução estática para o universo, já não era mais necessária a introdução da constante cosmológica.

Na década de 20, Friedman [3], e posteriormente Robertson [4] e Walker [5], propuseram uma solução às equações originais de Einstein considerando 
um universo preenchido uniformemente por matéria e radiação e que é completamente homogêneo e isotrópico em escalas da ordem de $10^{8}$ parsecs ou maiores. Neste caso a métrica tem a mesma forma em qualquer tempo, em qualquer ponto e em qualquer direção do espaço-tempo. Esta métrica ficou conhecida como a métrica de Friedmann-Robertson-Walker, que pode ser escrita como

$$
d s^{2}=-d t^{2}+a^{2}(t) R_{0}^{2}\left[\frac{d r^{2}}{1-k r^{2}}+r^{2} d \Omega^{2}\right]
$$

onde $d \Omega^{2}=d \theta^{2}+\operatorname{sen}^{2} \theta d \phi^{2}$ e $a(t)$ é o fator de escala que caracteriza o tamanho das seções espaciais, sendo dado, na sua forma normalizada, por $a(t)=\frac{R(t)}{R_{0}}$, em que $R_{0}$ correspondente ao tempo presente.

Vemos que nesta solução temos coordenadas comóveis em que o universo expande ou contrai de acordo com $R(t)$ porém as galáxias continuam com coordenadas fixas $r, \theta, \phi$.

Temos 3 tipos de universo descritos por esta solução, que correspondem aos parâmetros de curvatura $k$ iguais a $+1,0$ e -1 . Eles descrevem respectivamente um universo com curvatura positiva (quando a densidade de matéria e energia do universo é maior que a densidade crítica), um universo plano (com densidade crítica) e um universo com curvatura negativa (quando a densidade é menor que a densidade crítica).

No caso de um universo homogêneo e isotrópico, como o proposto por Friedmann, Robertson e Walker na solução acima, a fonte de energia e momento na equação de Einstein pode ser modelada como um fluido perfeito, que é um fluido homogêneo e isotrópico sem fricção e condução de calor, caracterizado por uma densidade de energia $\rho$ e uma pressão isotrópica $p$ no referencial de repouso. O tensor energia-momento de tal fluido pode ser escrito como [6]

$$
T_{\mu \nu}=(\rho(t)+p(t)) U_{\mu} U_{\nu}+p(t) g_{\mu \nu},
$$

onde $U^{\mu}$ é a quadrivelocidade do fluido.

O sistema de repouso do fluido deve ser o de um observador comóvel na métrica. Neste sistema as componentes tri-dimensionais da quadrivelocidade são iguais a zero $\left(u^{\alpha}=0, \alpha=1,2,3\right)$. Usando a métrica de FriedmannRobertson-Walker, o tensor energia-momento é escrito neste sistema de repouso como 


$$
T_{\mu \nu}=\left[\begin{array}{cccc}
-\rho & 0 & 0 & 0 \\
0 & a^{2} p & 0 & 0 \\
0 & 0 & a^{2} p & 0 \\
0 & 0 & 0 & a^{2} p
\end{array}\right] .
$$

Podemos contrair os índices utilizando a métrica, obtendo assim

$$
g^{\mu \nu} T_{\mu \nu}=T_{\mu}^{\mu}=T=\rho+3 p .
$$

Para um fluido não interagente temos que $\nabla_{\mu} T^{\mu \nu}=0$, pela conservação do tensor energia-momento. Calculando o divergente obtemos

$$
\dot{\rho}+3 H(\rho+p)=0
$$

que é a equação de conservação do fluido.

Se calcularmos, para a métrica de Friedmann-Robertson-Walker, os símbolos de Christoffel, as componentes do tensor de Ricci, a curvatura escalar e o traço do tensor energia-momento, e substituirmos na equação de Einstein, obtemos a seguinte equação para as componentes de índice ii

$$
\frac{\ddot{a}}{a}=-\frac{4 \pi G}{3}(\rho+3 p)
$$

e para as componentes de índice 00,

$$
\left(\frac{\dot{a}}{a}\right)^{2}=H^{2}=\frac{8 \pi G}{3} \rho-\frac{k}{a^{2} R_{0}^{2}},
$$

onde $H$ é o parâmetro de Hubble, que descreve a expansão do universo.

As equações (12) e (13) acima são conhecidas como equações de Friedman e junto com a métrica de Friedman-Robertson-Walker constituem o modelo padrão da cosmologia. O modelo de de Sitter corresponde ao caso especial do modelo de Friedman em que $k=0$ e $\rho=0$.

Vemos a partir das equações de Friedman que, para se ter um universo em expansão neste modelo, não é necessária, obrigatoriamente, a constante cosmológica. Porém vemos pela equação (12) que, para que esta expansão seja acelerada, é necessária a introdução da constante cosmológica, ou a introdução de uma componente de matéria que tenha pressão negativa.

De acordo com a equação (12) vemos que uma expansão acelerada ocorre sempre que $\rho+3 p<0$. De acordo com a equação de estado dos fluidos, postulada como sendo 


$$
p=w \rho,
$$

onde $w$ é uma constante fenomenológica, temos uma expansão acelerada quando

$$
w<-\frac{1}{3}
$$

Também podemos escrever (12) na forma

$$
\Omega(t)-1=\frac{k}{a^{2} H^{2}},
$$

onde $\Omega(t)$ é o parâmetro de densidade total dado por

$$
\Omega(t)=\frac{\rho(t)}{\rho_{c}},
$$

sendo $\rho_{c}$ a densidade crítica necessária para termos um universo plano (o que corresponde a termos $k=0$ ). Esta densidade crítica pode ser obtida a partir da equação (13). Considerando $k=0$ e isolando $\rho$ obtém-se

$$
\rho_{c}(t)=\frac{3 H^{2}(t)}{8 \pi G}
$$

Atualmente as observações da Radiação Cósmica de Fundo apontam para um universo plano, ou seja com $k=0$ [7]. Sabe-se também que a densidade crítica necessária para um universo plano é da ordem de

$$
\rho_{c}=m_{p l}^{2} H_{0}^{2} \sim 10^{-47} G e V^{4} .
$$

No entanto apenas $30 \%$ deste valor é observado direta ou indiretamente em forma de matéria, $25 \%$ correspondendo à matéria escura e $5 \%$ à matéria bariônica.

Temos hoje evidências fortes de que o universo está se expandindo aceleradamente. Diversos experimentos baseados em fenômenos distintos como medidas de supernovas, medidas da Radiação Cósmica de Fundo, entre outros, apontam para o mesmo resultado [7]. Devido a estas observações a introdução da constante cosmológica nas equações de Einstein voltou a ser um assunto em questão . Como vimos, para termos uma expansão acelerada, de acordo com a teoria da relatividade geral, é necessário introduzirmos uma 
constante cosmológica, ou um fluido com pressão negativa, que tem o mesmo efeito repulsivo da constante cosmológica. É possível que esta componente responsável pela expansão do universo, chamada hoje de energia escura, possa ser a responsável pelos $70 \%$ de energia faltantes no universo. Seja o que for esta componente, acredita-se que ela deva ter um comportamento não muito diferente de uma constante cosmológica, pois os dados atuais são compatíveis com uma energia escura com equação de estado constante $w=-1$ e densidade de energia da ordem de $10^{-47} \mathrm{GeV}^{4}$, dando margem porém á pequenas variações em torno destes valores.

Com a inclusão do termo da constante cosmológica as equações de Friedman se tornam

$$
\begin{aligned}
& \frac{\ddot{a}}{a}=-\frac{4 \pi G}{3}(\rho+3 p)+\frac{\Lambda}{3}, \\
& H^{2}=\frac{8 \pi G}{3} \rho-\frac{k}{a^{2} R_{0}^{2}}+\frac{\Lambda}{3} .
\end{aligned}
$$

Se introduzirmos as seguintes expressões para as densidades de energia e pressão modificadas,

$$
\begin{aligned}
& \widetilde{\rho}=\rho+\frac{\Lambda}{8 \pi G} \\
& \widetilde{p}=p-\frac{\Lambda}{8 \pi G}
\end{aligned}
$$

podemos ver que as equações acima se reduzem às equações (12) e (13).

Os modelos de quintessência [8] são exemplos de modelos nos quais o fluido com pressão negativa corresponde a um campo escalar com potencial não nulo e equação de estado aproximadamente $w=-1$. Há também tentativas de se associar a constante cosmológica à energia do vácuo, mas nenhuma até agora obteve êxito devido à grande diferença na ordem de magnitude da energia do vácuo prevista pela teoria de campos e da energia esperada para a constante cosmológica. Outra possibilidade ainda é alterar o lado esquerdo da equação de Einstein, ou seja, alterar a própria teoria da relatividade geral, mudando a forma como a geometria do espaço-tempo reage à presença de matéria e energia, e neste caso a Relatividade Geral seria apenas uma teoria efetiva à baixas energias. Teorias como esta são chamadas teorias de gravidade modificada. Um exemplo são as chamadas teorias $f(R)$. 
Nestas teorias a função densidade lagrangeana $f(R)$ é uma função arbitrária do escalar de curvatura $R[13,14,15,16]$.

As possibilidades mencionadas acima serão discutidas nos próximos capítulos.

\subsection{1 - A Constante Cosmológica}

Um dos problemas relacionados à constante cosmológica se refere à interpretação física da origem de sua energia. Considere um campo escalar $\phi$ com um potencial $\mathrm{V}(\phi)$. Sua ação é dada pela expressão

$$
S=\int d^{4} x \sqrt{-g}\left[\frac{1}{2} g^{\mu \nu} \partial_{\mu} \phi \partial_{\nu} \phi-V(\phi)\right]
$$

onde $g$ é o determinante da métrica.

Sabemos que o tensor energia-momento deste campo é definido como

$$
T_{\mu \nu}=\frac{-2}{\sqrt{-g}} \frac{\delta S}{\delta g^{\mu \nu}} .
$$

A partir desta expressão obtemos para o tensor energia-momento

$$
T_{\mu \nu}=\partial_{\mu} \phi \partial_{\nu} \phi+g_{\mu \nu} L
$$

onde $L$ é a Lagrangeana do campo escalar.

No estado de vácuo de um campo escalar, sua energia cinética é nula, porém sua energia potencial pode ser diferente de zero. Neste caso o tensor energia momento do campo é

$$
T_{\mu \nu}=-V\left(\phi_{0}\right) g_{\mu \nu},
$$

sendo $\phi_{0}$ o valor de $\phi$ que minimiza este potencial. Podemos então escrever o tensor energia-momento deste campo como

$$
T_{\mu \nu}^{v a ́ c}=-\rho_{v a ́ c} g_{\mu \nu} .
$$

Neste estado de vácuo o campo tem equação de estado correspondendo a $w=-1$. O efeito do tensor energia-momento acima é o mesmo que o de uma constante cosmológica. Isto pode ser visto claramente se mudarmos o termo $\Lambda g_{\mu \nu}$ na equação de Einstein para o lado direito e igualarmos à densidade de energia do vácuo, 


$$
\rho_{\text {vácuo }}=\rho_{\Lambda}=\frac{\Lambda}{8 \pi G} .
$$

Este é o motivo da associação da constante cosmológica à energia do vácuo. Neste contexto a constante cosmológica poderia ter contribuições advindas das energias dos campos escalares nos mínimos de seus potenciais, ou seja, nos seus estados de vácuo, e também das flutuações de vácuo de cada grau de liberdade das teorias de campos [6].

De acordo com o princípio da incerteza sabemos que o estado de mínima energia de um campo escalar não tem energia nula. Um campo quântico pode ser interpretado como um número infinito de osciladores harmônicos no espaço dos momentos. Portanto a energia de vácuo de todos estes osciladores deveria se somar á infinito, pois a mesma pode ser calculada integrando-se no espaço dos momentos todas as contribuições [8]

$$
\begin{gathered}
\rho_{\text {vác }}=\frac{1}{2} \int_{0}^{\infty} \frac{d^{3} k}{(2 \pi)^{3}} \sqrt{k^{2}+m^{2}} \\
=\frac{1}{4 \pi^{2}} \int_{0}^{\infty} d k k^{2} \sqrt{k^{2}+m^{2}} .
\end{gathered}
$$

No entanto, podemos renormalizar nossa teoria, descartando todos os modos correspondentes a altas energias (ou altos momentos), pois não conhecemos a nossa teoria nessa escala de energia, então não sabemos mais se ela é válida nesse domínio [17]. Ao fazermos um corte no momento máximo no qual integramos nossa teoria obtemos uma densidade de energia do vácuo com a forma [8]

$$
\rho_{\text {vácuo }} \propto k_{\text {máx }}^{4}
$$

$\mathrm{Na}$ ausência de gravidade poderíamos alterar esta energia somando e diminuindo termos como quiséssemos, mas com a gravidade é preciso considerar corretamente esta energia.

No caso das flutuações de vácuo, para que a densidade de energia não vá para infinito, consideramos um corte na integral na escala de $m_{p}=10^{19} \mathrm{GeV}$, pois supomos que nossa teoria quântica de campos seja válida até esta escala [8]. Neste caso, obtemos a seguinte contribuição para a energia de vácuo devido às flutuações quânticas dos campos, 


$$
\rho_{\text {vácuo }}=\left(10^{19} \mathrm{GeV}\right)^{4} \sim 10^{74} \mathrm{GeV}^{4},
$$

que é $10^{121}$ ordens de magnitude maior que o valor observado da constante cosmológica.

Outras contribuições que poderiam ser associadas a uma constante cosmológica são as energias do mínimo do potencial dos campos escalares. A energia $V(\phi)$ dos campos é uma grandeza que varia com o tempo à medida em que o universo passa por transições de fase. Quando o universo se esfria os campos tendem a assumir seus estados de vácuo, com energia cinética nula, porém, com um potencial cujo mínimo não necessariamente é igual a zero. E este valor do potencial diferente de zero poderia então contribuir para a energia do vácuo [6].

$\mathrm{Na}$ teoria eletrofraca de Weinberg-Salam a fase da simetria eletrofraca quebrada difere da fase simétrica por uma energia potencial da ordem de $M_{e w}=200 \mathrm{GeV}$. Acredita-se que o universo esteve na fase simétrica em seus momentos iniciais quando a temperatura era muito alta. Com a diminuição da temperatura o universo sofreu uma mudança de fase. A contribuição para a energia de vácuo é então diferente nas duas fases e podemos estimar a diferença entre estas densidades de energia como sendo [6]

$$
\rho_{\text {vácuo }}^{E W} \sim(200 G e V)^{4} .
$$

Já no caso da QCD por exemplo, acredita-se que a simetria quiral seja quebrada por um valor esperado de vácuo não nulo do condensado de quarks $\bar{q} q$. Neste caso a diferença de energia entre a fase quebrada e a fase simétrica é da ordem de $M_{Q C D} \sim 0,3 G e V$. Então teremos uma contribuição à densidade de energia do vácuo da ordem de

$$
\rho_{\text {vácuo }}^{Q C D} \sim(0,3 G e V)^{4} .
$$

Além destas contribuições poderíamos acrescentar diversas outras contribuições advindas de transições de fase no universo primordial, no entanto, estas contribuições, assim como as mencionadas acima, possuem todas, isoladamente, ordens de magnitude bem superiores ao valor observado da constante cosmológica, que é $10^{-47} \mathrm{GeV}^{4}$ [8].

Não há nenhum motivo que proíba que todas estas contribuições independentes, com sinais positivos e negativos, se somem para gerar o valor observado atualmente da constante cosmológica. Entretanto, o ajuste fino 
necessário para isto seria enorme. Isto faz com que talvez seja mais natural pensar que haja algum mecanismo desconhecido que anule estas contribuições do que pensar que elas se somam para resultar em um valor tão pequeno como o observado. Neste caso a energia escura poderia estar associada a algum outro campo escalar desconhecido que forneceria a densidade de energia que observamos. Esta idéia corresponde ao modelo da quintessência que será discutido a seguir. 


\section{2 - Quintessência}

Vimos que, de acordo com a equação de Friedmann (12), uma expansão acelerada ocorre no universo sempre que ele for dominado por um fluido cuja equação de estado, dada por $p=w \rho$, tenha o parâmetro $w$ seguindo a

condição $w<-\frac{1}{3}$. Chamamos de quintessência um campo escalar que possui uma equação de estado desse tipo, e que portanto poderia ser responsável pela atual aceleração do universo.

A ação da quintessência é dada por [8]

$$
S=\int d^{4} x \sqrt{-g}\left[-\frac{1}{2} \partial_{\mu} \phi \partial^{\mu} \phi-V(\phi)\right]
$$

Podemos obter a equação de movimento para este campo minimizando a ação. Isto nos fornecerá a equação de movimento no espaço-tempo plano de Friedmann-Robertson-Walker,

$$
\ddot{\phi}+3 H \dot{\phi}+\frac{d V(\phi)}{d \phi}=0 .
$$

Ver demonstração no Apêndice A.

Vimos que o tensor energia-momento de um campo escalar é dado pela expressão (26). Subindo um índice do tensor energia-momento e considerando um espaço-tempo homogêneo e plano, obtemos as seguintes expressões para as densidades de pressão e energia do campo escalar,

$$
\begin{gathered}
\rho=-T_{0}^{0}=\frac{1}{2} \dot{\phi}^{2}+V(\phi), \\
p=T_{i}^{i}=\frac{1}{2} \dot{\phi}^{2}-V(\phi) .
\end{gathered}
$$


Então a equação de estado do campo $\phi$ é dada por

$$
w_{\phi}=\frac{p}{\rho}=\frac{\frac{1}{2} \dot{\phi}^{2}-V(\phi)}{\frac{1}{2} \dot{\phi^{2}}+V(\phi)} .
$$

Podemos ver que quando $\phi \ll V(\phi)$, a equação de estado é $w=-1$, o que corresponde à equação de estado da constante cosmológica. Quando $\phi \gg V(\phi)$, vemos que $w=1$. Quando a pressão é igual a zero temos a equação de estado $w=0$.

Substituindo as expressões de $\rho$ e $p$ nas equações de Friedmann no espaçotempo plano obtemos

$$
\begin{aligned}
& H^{2}=\frac{8 \pi G}{3}\left[\frac{1}{2} \dot{\phi}^{2}+V(\phi)\right], \\
& \ddot{a}=-\frac{8 \pi G}{3}\left[\dot{\phi}^{2}-V(\phi)\right] .
\end{aligned}
$$

No caso de um universo dominado por um fluido com equação de estado constante, a evolução do universo pode ser descrita através das equações

$$
\begin{gathered}
H=\frac{2}{3(1+w)\left(t-t_{0}\right)}, \\
a(t) \propto\left(t-t_{0}\right)^{\frac{2}{3(1+w)}}, \\
\rho \propto a^{-3(1+w)} .
\end{gathered}
$$

onde $t_{0}$ é uma constante. Vemos que as soluções acima são válidas para $w \neq-1$.

Em um universo dominado por radiação $w=\frac{1}{3}$. Neste caso vemos que $a(t) \propto\left(t-t_{0}\right)^{\frac{1}{2}}$ e $\rho \propto a^{-4}$.

Em um universo dominado por matéria, $w=0$. Neste caso $a(t) \propto\left(t-t_{0}\right)^{\frac{2}{3}}$ e $\rho \propto a^{-3}$.

Em um universo dominado por uma energia escura tal que $w<-\frac{1}{3}$, temos uma expansão do tipo potência, $a(t) \propto\left(t-t_{0}\right)^{p}$, sendo $p>1$.

No caso da constante cosmológica, $w=-1$. Neste caso $\rho$ é uma constante. Resolvendo as equações de Friedmann para este caso obtemos uma expansão exponencial do tipo $a(t) \propto e^{H t}$. 
A fim de descrever a expansão acelerada do universo, diversos modelos de energia escura baseados em campos escalares foram propostos. Originalmente os modelos de quintessência foram descritos por potenciais do tipo [8],

$$
V(\phi)=\frac{M^{(4+\alpha)}}{\phi^{\alpha}},
$$

onde $\alpha$ pode ser positivo e também negativo.

Um exemplo de um potencial do tipo potência foi também proposto por Friedmann em 1995 [9], que correspondia a

$$
V(\phi)=\frac{1}{2} m^{2} \phi^{2}+\frac{1}{4 !} \lambda \phi^{4}
$$

onde $m^{2}$ pode também ser menor que zero.

Em modelos em que a energia escura é descrita por um campo escalar é necessário que, caso hajam acoplamentos com as partículas do modelo padrão, eles sejam muito fracos, caso contrário este campo já haveria sido observado.

Potenciais do tipo exponencial são também capazes de gerar a expansão acelerada que observamos. Podemos citar alguns modelos deste tipo, como $[10,11,12]$

$$
\begin{gathered}
V(\phi)=K e^{-\phi \sqrt{8 \pi q G}}, \\
V(\phi)=K \phi^{-\alpha} e^{\lambda \phi^{2}}, \\
V(\phi)=K\left(e^{\alpha k \phi}+e^{\beta k \phi}\right),
\end{gathered}
$$

entre outros. Nestes modelos a densidade do campo escalar passa a dominar apenas no presente, quando a forma plana do potencial garante uma equação de estado para o campo compatível com um universo com a expansão que observamos. É necessário também que a densidade de energia do campo corresponda à densidade crítica $\rho=10^{-47} \mathrm{GeV}$. Diversos outros modelos baseados em idéias como estas já foram propostos, sendo também capazes de explicar os dados observacionais que temos hoje.

Um modelo em que a energia escura é interpretada como um campo escalar, precisa, para estar de acordo com os dados observacionais, que este campo de alguma forma se comporte de modo semelhante a uma constante 
cosmológica, ou seja, tenha energia cinética igual ou muito próxima de zero e energia potencial da ordem de grandeza de $10^{-47} \mathrm{GeV}$. Este campo possui equação de estado dada aproximadamente por $w \simeq-1$. Mas um modelo que reproduza exatamente a constante cosmológica sofreria dos mesmos problemas que esta, como o problema da coincidência, por exemplo, o problema da idade de algumas estruturas antigas no universo serem incompatíveis com as previsões do Modelo Padrão [18], entre outros. No entanto a medida do parametro $w$ possui uma acurácia de $5 \%$ a $10 \%$ apenas [19], e por isto é possível termos um $w$ efetivo que possui correções ao valor $w=-1$. Uma situação em que temos este cenário é quando há uma troca de energia entre a energia escura e um outro setor do universo, como a matéria escura por exemplo. Este modelo é o chamado modelo interagente e veremos a seguir como ele pode resolver ou amenizar os problemas mencionados. 


\section{3- A Interação no Setor Escuro do Universo}

As observações atuais apontam que a energia escura compõe $70 \%$ do conteúdo energético do universo, e a matéria escura aproximadamente $25 \%$. Vimos que existem diversos modelos para descrever o setor escuro do universo, entre eles os que tratam estas componentes como campos escalares. No caso destes modelos é natural supor que, sendo estes dois campos os responsáveis pela maior parte do conteúdo energético do universo, haja uma interação entre eles. A possibilidade de haver uma interação entre a matéria e a energia escura tem sido muito discutida recentemente [20, 21, 22, 23, 24, 25]. Nestes modelos de interação supõe-se que haja uma troca de energia entre estes dois setores do universo. Neste caso a energia e a matéria escura não se conservam mais separadamente. As equações de estado das mesmas são alteradas ficando então, respectivamente, na forma

$$
\begin{gathered}
\rho_{E}^{\prime}=-3 H\left(1+w_{E}\right) \rho_{E}+a Q_{E}, \\
\rho_{M}^{\prime}=-3 H\left(1+w_{M}\right) \rho_{M}+a Q_{M},
\end{gathered}
$$

onde $Q_{e}=-Q_{m}$ é a taxa de transferência de energia, e a apóstrofe indica derivada com relação ao tempo conforme $\tau$.

Podemos definir um $w$ efetivo para a energia escura da forma

$$
w_{e f}=w-\frac{a Q_{E}}{3 H \rho_{E}} .
$$

Se supusermos, por exemplo, um decaimento de energia escura em matéria escura é de se esperar que o fator $Q$ seja proporcional à densidade de energia da energia escura e à sua taxa de decaimento. Neste caso temos 


$$
Q_{e}=-Q_{m}=-\Gamma \rho_{e}
$$

A solução de (51) é dada por

$$
\rho_{E}=\rho_{E 0} a^{-3(1+w)} e^{-\Gamma\left(t-t_{0}\right)} .
$$

Ao se considerar, nas equações cosmológicas, esta troca de energia, foi visto que diversas previsões são alteradas com relação ao modelo não interagente, algumas das quais podem vir a ser observadas. Com este modelo viu-se, por exemplo, que para determinados valores do acoplamento é possível explicar como certos quasares muito antigos podem ter evoluído ao longo do tempo de vida do universo. [26].

É possível mostrar também que a interação no setor escuro do universo pode deixar marcas nos aglomerados de galáxias devido á uma correção no processo de virialização do aglomerado causado pela interação [27]. Com este efeito os parâmetros do modelo podem ser testados.

Mais recentemente foi observado um desalinhamento entre a matéria escura e a matéria bariônica nos aglomerados de galáxias, o que está em desacordo com as previsões do modelo padrão [28]. Tal desalinhamento foi observado através do efeito de lentes gravitacionais. Com a suspeita de que este efeito pudesse ser causado por uma interação entre a matéria e a energia escura foram feitos trabalhos baseados em simulações que apontam que a interação de fato pode aumentar o desalinhamento com relação ao previsto pelo Modelo Padrão [29, 30].

Além disso, a interação é capaz de aliviar o problema da coincidência na cosmologia, que vem do fato de que não esperamos estar em uma situação especial ou rara na história do universo. Como a matéria escura e a energia escura decaem com a expansão do universo a diferentes taxas, surge a seguinte questão: "Por que a densidade da energia e da matéria escura são da mesma ordem de grandeza justamente hoje se durante toda a evolução do universo elas tiveram ordens de grandeza tão distintas?" Com uma interação entre estes dois setores, ou um decaimento de um deles no outro, cada componente passa a não ser mais separadamente conservada, como vimos acima. Isto faz com que ambas as componentes evoluam de forma correlacionada [31].

Com um acoplamento adequado entre a energia e a matéria escura é possível reproduzir uma solução de escala do tipo [32]

$$
\rho_{E} \propto \rho_{M} a^{\xi}
$$


Para a equação de estado do modelo $\Lambda C D M, p_{E}=-\rho_{E}$, temos $\xi=3$ em qualquer instante de tempo. Um valor $\xi=0$ representa a razão $\frac{\rho_{M}}{\rho_{E}}=$ cte. Se a dinâmica cosmológica admite uma solução estacionária com esta razão, que corresponde a $\xi=0$, e o universo atual está próximo a esta solução, então não há problema de coincidência [33]. Portanto a variação do parâmetro $\xi$ com relação a zero quantifica a gravidade do problema. Neste caso qualquer solução que se desvie de $\xi=-3 w_{E}$, que tenha $\xi<3$ alivia o problema da coincidência, como de fato ocorre com o modelo mostrado anteriormente em que a energia escura decai em matéria escura. Isto pode ser visto claramente através da nova equação de $w_{\text {ef }}$ (53). Portanto, vemos que o modelo interagente é capaz de amenizar este problema.

Diversas análises já foram feitas usando dados de WMAP, SNIa, BAO e SDSS, dando indícios da interação [26]. Foram analisadas consequências da interação no crescimento de estruturas [24, 25], e também correções observáveis na equação de Layser-Irvine [27]. Como consequência temos hoje boas evidências de um acoplamento pequeno indicando uma transferência de energia escura para matéria escura, o que alivia o problema da coincidência e está de acordo com os resultados de CMB [22]. 


\section{4 - Gravitação Modificada}

Vimos que é possível descrever uma expansão acelerada no universo ao se acrescentar fontes de energia, com equação de estado $w<-\frac{1}{3}$, ao tensor energia-momento na equação de Einstein. Outra possibilidade no entanto é alterar o lado esquerdo da equação modificando a própria teoria da gravitação, ou seja, alterando a forma como a geometria do espaço-tempo reage à presença da matéria. Sabemos que a Teoria da Relatividade Geral é uma teoria que concorda muito bem com os dados observacionais que temos hoje, no entanto há limites em que ela ainda não foi testada. Modificações geométricas à Relatividade Geral poderiam advir de efeitos quânticos, como correções à ação de Einstein-Hilbert devido à efeitos de grandes curvaturas [8].

A densidade Lagrangeana na Relatividade Geral é dada por $\mathrm{f}(R)=R-2 \Lambda$. Uma forma de modificar a Relatividade Geral é considerar uma densidade Lagrangeana como sendo uma função arbitrária de $R$. Não é fácil porém, encontrar uma função $f(R)$ que descreva a atual aceleração do universo, não possua instabilidades e satisfaça os vínculos observacionais. Para estar de acordo com estes vínculos é necessário que no limite local, em regiões de densidade superior ao background, estas teorias recuperem a Relatividade Geral. Algumas teorias com estas características [34, 35, 36, 37] são atualmente uma alternativa viável para se explicar o comportamento atual do universo.

Na teoria de gravitação $f(R)$ as previsões a longas distâncias diferem da Relatividade Geral, e alguns sinais destas diferenças podem vir a ser observados. Estas diferenças poderiam causar certas modificações no espectro de aglomerados de galáxias [38, 39], poderiam também deixar marcas na CMB [40, 41] e causar alterações em efeitos de lentes gravitacionais [42, 43]. Tais efeitos podem vir a ser um indicativo de uma nova descrição da gravitação. 
A Lagrangeana 4-dimensional numa teoria $f(R)$ é dada no referencial de Jordan por

$$
S=\frac{1}{2 k^{2}} \int d^{4} x \sqrt{-g} f(R)+\int d^{4} x L^{(m)},
$$

onde $R$ é o escalar de Ricci, $k^{2}=8 \pi G$ e $L^{(m)}$ é a Lagrangeana da matéria.

Variando esta ação com relação à métrica $g_{\mu \nu}$, dentro do formalismo padrão da métrica, obtemos a seguinte equação de campo

$$
F R_{\mu \nu}-\frac{1}{2} f g_{\mu \nu}-\nabla_{\mu} \nabla_{\nu} F+g_{\mu \nu} \square F=k^{2} T_{\mu \nu}^{(m)}
$$

onde $F=\frac{\partial f}{\partial R}$. Quando $f(R)=R$ esta equação se reduz à Equação de Einstein.

A equação acima pode ser reescrita na forma

$$
G_{\mu \nu}=\frac{1}{2 F}(f-R F) g_{\mu \nu}+\frac{1}{F}\left(\nabla_{\mu} \nabla_{\nu}-g_{\mu \nu} \square\right) F+k^{2} \frac{T_{\mu \nu}^{(m)}}{F},
$$

onde $G_{\mu \nu}=R_{\mu \nu}-\frac{1}{2} R g_{\mu \nu}$.

Se definirmos o tensor energia-momento efetivo como

$$
T_{\mu \nu}^{(e)}=\frac{1}{2 k^{2}}(f-R F) g_{\mu \nu}+\frac{1}{k^{2}}\left(\nabla_{\mu} \nabla_{\nu}-g_{\mu \nu} \square\right) F
$$

podemos reescrever as equações de campo como

$$
G_{\mu \nu}=\frac{k^{2}}{F}\left(T_{\mu \nu}^{(e)}+T_{\mu \nu}^{(m)}\right) .
$$

Podemos discutir a gravitação $f(R)$ no referencial de Einstein fazendo a seguinte transformação

$$
\tilde{g}_{\mu \nu}=\Omega^{2} g_{\mu \nu},
$$

onde $\Omega^{2}$ é o fator conforme e representa as coordenadas no referencial de Einstein. O escalar de Ricci nos dois referenciais se relacionam através da equação

$$
R=\Omega^{2}\left(\tilde{R}+6 \tilde{\square} w-6 \tilde{g}^{\mu \nu} \partial_{\mu} w \partial_{\nu} w\right),
$$

onde $w=\ln \Omega, \partial_{\mu} w=\frac{\partial w}{\partial \tilde{x}^{\mu}}, \tilde{\square} w=\frac{1}{\sqrt{-\tilde{g}}} \partial_{\mu}\left(\sqrt{-\tilde{g}} \tilde{g}^{\mu \nu} \partial_{\nu} w\right)$.

Podemos escrever a ação original na forma 


$$
S=\int d^{4} x \sqrt{-g}\left(\frac{1}{2 k^{2}} F R-U\right)+\int d^{4} x L^{(m)}\left(g_{\mu \nu}, \Psi_{M}\right),
$$

onde $U=\frac{F R-f}{2 k^{2}}$. Usando a expressão do escalar de Ricci e a relação $\sqrt{-g}=$ $\Omega^{-4} \sqrt{-\tilde{g}}$, a ação acima pode ser escrita como

$$
S=\int d^{4} x \sqrt{-\tilde{g}}\left[\frac{1}{2 k^{2}} F \Omega^{-2}\left(\tilde{R}+6 \tilde{\square} w-6 \tilde{g}^{\mu \nu} \partial_{\mu} w \partial_{\nu} w\right)-\Omega^{-4} U\right]+\int d^{4} x L^{(m)}\left(\Omega^{-2} \tilde{g}_{\mu \nu}, \Psi_{M}\right),
$$

obtemos a ação no referencial de Einstein para a escolha $\Omega^{2}=F($ para $F>0)$ - Podemos introduzir um campo escalar definindo

$$
k \phi=\sqrt{\frac{3}{2}} \ln F .
$$

Como $w=\ln \Omega$, temos que $w=\frac{k \phi}{\sqrt{6}}$. O termo na integral da ação proporcional a $\tilde{\square} w$ zera devido ao teorema de Gauss. Portanto, a ação no referencial de Einstein fica

$$
S=\int d^{4} x \sqrt{-\tilde{g}}\left[\frac{1}{2 k^{2}} \tilde{R}-\frac{1}{2} \tilde{g}^{\mu \nu} \partial_{\mu} \phi \partial_{\nu} \phi-V(\phi)\right]+\int d^{4} x L^{(m)}\left(F^{-1}(\phi) \tilde{g}_{\mu \nu}, \Psi_{M}\right),
$$

onde $V(\phi)=\frac{U}{F^{2}}=\frac{F R-f}{2 k^{2} F^{2}}$, e $-\frac{1}{2} \tilde{g}^{\mu \nu} \partial_{\mu} \phi \partial_{\nu} \phi-V(\phi)=L_{\phi}$ é a Lagrangeana do campo escalar. Podemos ver pela Lagrangeana da matéria que a mesma é acoplada ao campo escalar.

Podemos derivar o tensor energia-momento do campo escalar

$$
\tilde{T}_{\mu \nu}^{(\phi)}=\frac{-2}{\sqrt{-\tilde{g}}} \frac{\delta\left(\sqrt{-\tilde{g}} L_{\phi}\right)}{\delta \tilde{g}^{\mu \nu}}=\partial_{\mu} \phi \partial_{\nu} \phi-\tilde{g}_{\mu \nu}\left[\frac{1}{2} \tilde{g}^{\alpha \beta} \partial_{\alpha} \phi \partial_{\beta} \phi+V(\phi)\right]
$$

Variando a ação total no referencial de Einstein com relação ao campo $\phi$ obtemos

$$
-\partial_{\mu}\left(\frac{\partial\left(\sqrt{-\tilde{g}} L_{\phi}\right)}{\partial\left(\partial_{\mu} \phi\right)}\right)+\partial\left(\frac{\partial\left(\sqrt{-\tilde{g}} L_{\phi}\right)}{(\partial \phi)}\right)+\frac{\partial L^{(m)}}{\partial \phi}=0
$$

que nos fornece a equação de movimento

$$
\tilde{\square} \phi-V_{, \phi}+\frac{1}{\sqrt{-\tilde{g}}} \frac{\partial L^{(m)}}{\partial \phi}=0,
$$


onde $\tilde{\square} \phi=\frac{1}{\sqrt{-g}} \partial_{\mu}\left(\sqrt{-\tilde{g}} \tilde{g}^{\mu \nu} \partial_{\nu} \phi\right)$. Variando a Lagrangeana da matéria com relação a $\phi$ obtemos

$$
\frac{\partial L^{(m)}}{\partial \phi}=\frac{1}{F(\phi)} \frac{\partial L^{(m)}}{\partial \tilde{g}^{\mu \nu}} \frac{\partial\left(F(\phi) \tilde{g}^{\mu \nu}\right)}{\partial \phi}=-\sqrt{-\tilde{g}} \frac{F_{, \phi}}{2 F} \tilde{T}_{\mu \nu}^{(m)} \tilde{g}^{\mu \nu},
$$

e então,

$$
\tilde{\square} \phi-V_{, \phi}+k Q \tilde{T}=0,
$$

onde $Q=-\frac{F_{, \phi}}{2 k F}$ e $\tilde{T}=\tilde{g}_{\mu \nu} \tilde{T}^{\mu \nu(m)}$.

Podemos ver que o modelo de gravitação modificada $f(R)$ equivale, sob uma transformação conforme, a um modelo de quintessência com interação, e é portanto capaz de explicar a atual expansão acelerada do universo. É possível mostrar que a condição para que a teoria $f(R)$ não possua instabilidades no universo primordial e esteja de acordo com as observações de CMB equivale à condição de que haja uma transferência de energia partindo da energia escura para outros setores do universo, o que, como vimos no capítulo anterior, ajuda a amenizar o problema da coincidência [44]. 


\section{5 - Um Modelo para a Energia Escura}

Nos capítulos anteriores vimos que a constante cosmológica possui boa concordância com os resultados experimentais. Vimos também que apesar do modelo padrão $\Lambda C D M$ se ajustar muito bem às observações ele possui certos problemas que podem ser melhor explicados por outros modelos, como o modelo de gravidade $f(R)$ ou o modelo de interação, por exemplo. Buscamos então, no presente trabalho, um modelo baseado em teoria de campos que pudesse descrever um mecanismo para o decaimento da energia escura em matéria escura, e que ao mesmo tempo se aproximasse do comportamento de uma constante cosmológica. Uma possibilidade neste contexto é representar a energia escura por um campo escalar situado em um mínimo metaestável de um potencial. Modelos baseados nesta idéia foram considerados já na década de 80 e 90 em trabalhos como [45, 46, 47], no entanto, desconsiderando um possível decaimento do mínimo metaestável para o mínimo estável do potencial.

Supomos então no nosso modelo um campo escalar cujo potencial tem um duplo vácuo com diferença de energia de $10^{-47} \mathrm{GeV}^{4}$. Esta situação é bastante rara, exceto para um caso bem conhecido: quando há uma simetria forçando os dois vácuos a serem iguais, e uma quebra não perturbativa desta simetria. Um exemplo deste caso é o modelo supersimétrico de Wess-Zumino [48], cuja parte bosônica é dada pela equação

$$
U=\left|2 m \phi-3 \lambda \phi^{2}\right|^{2}+Q(\phi),
$$

onde $\phi=\varphi+i B$ e $Q(\phi)$ representa o termo de quebra de supersimetria que será ajustado de modo a ter o valor da constante cosmológica no segundo 
mínimo do potencial. Consideraremos um termo de quebra do tipo potência em $\phi$, cuja forma específica não será necessária para os cálculos. Consideraremos nos nossos cálculos apenas a parte real do campo, ou seja, $B=0$. Como vimos, um modelo de Einstein para a gravitação com uma interação bosônica real é equivalente a um modelo de gravidade $f(R)$. Desprezamos a interação fermiônica pois além de considerarmos como sendo muito pequena, ela será irrelevante para os cálculos em questão. O potencial deste modelo está representado na figura a seguir:

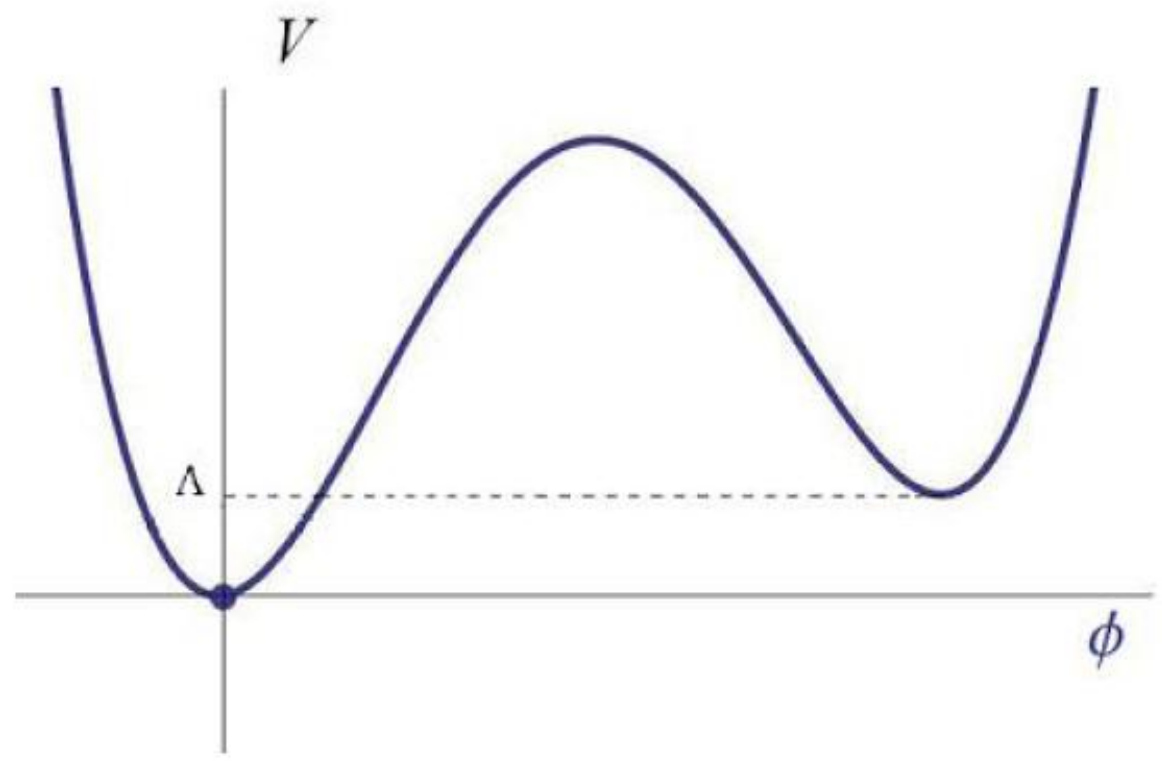

Figura 5.1: O potencial para a energia escura.

A quebra de supersimetria observada no modelo padrão corresponde a um valor maior que o valor representado pela constante cosmológica no nosso caso. Porém até hoje não se sabe exatamente como esta quebra ocorre e nem o mecanismo pelo qual ela é transmitida. Devemos lembrar também que como a energia escura até hoje nunca foi observada, supõe-se que ela possua interações muito fracas com as partículas do Modelo Padrão. Apesar de termos feito $B=0$ consideramos a possibilidade de que haja alguma simetria que explique um potencial com as características mencionadas, no entanto 
sabemos que um modelo de energia escura deste tipo, representado por um vácuo metaestável, pode ser viável independente deste tipo de interpretação.

Como o valor da constante cosmológica é muito pequeno é de se esperar que o tempo de decaimento da partícula de energia escura seja muito maior que a idade do universo. No entanto à medida que decrescemos o parâmetro massa da partícula de energia escura, a altura e a largura da barreira de seu potencial também decrescem, portanto esperamos que em um certo ponto a densidade de energia correspondente à constante cosmológica não corresponda mais à um ajuste fino muito grande. Então podemos esperar que haja um decaimento para valores muito pequenos de massa. Ao decair o campo passará a ter uma energia cinética e consequentemente sua equação de estado será alterada e ele passará a se comportar como matéria.

Atualmente, tem se estudado a possibilidade de haver partículas ultra leves que poderíam ser candidatas ao setor escuro do universo. Como as partículas candidatas ao setor escuro do universo nunca foram descobertas nos detectores de partículas, pensa-se que ou elas tenham massas muito grandes ou possuem interações muito fracas com as partículas conhecidas atualmente. Um exemplo deste último caso são os áxions [49, 50, 51], candidatos à matéria escura do universo, que caso existam, devem possuir massa na faixa de $10^{-2}$ a $10^{-5} \mathrm{eV}$ [52].

Os áxions são um dos mais antigos candidatos à partícula de matéria escura, e são ainda hoje um dos mais atrativos [52]. Além de resolver o problema de $\mathrm{CP}$ forte, o fato de serem um bom candidato à matéria escura abriu novas possibilidades relacionadas às características da partícula de matéria escura. Hoje sabemos que partículas ultra leves, na faixa de massa citada acima, podem ser boas condidatas à matéria escura e passam em todos os testes observacionais [52], desde que tenham interações fracas o suficiente para nunca terem sido observadas. Podemos então pensar que além dos áxions outros candidatos com características diferentes podem existir nesta faixa de massa.

No presente trabalho demonstraremos o decaimento de uma partícula de energia escura em matéria escura, o que pode fornecer um mecanismo para explicar a interação no setor escuro do universo partindo de teoria de campos. Para calcular o decaimento utilizaremos o método do cálculo de instantons e uma aproximação usada inicialmente por Coleman [53], o que nos permitirá obter uma expressão analítica para a taxa de decaimento da energia escura. Este método será descrito no capítulo a seguir. 


\section{6-O Método Semiclássico do Tunelamento}

Supomos uma partícula situada no mínimo metaestável de um potencial do tipo dado na figura 5.1 do capítulo anterior. Sabemos que de acordo com a mecânica quântica existe uma probabilidade não nula da partícula situada no mínimo metaestável tunelar a barreira de potencial e decair para o mínimo estável. Para que esta transição ocorra o campo correspondente à partícula em questão, deve atravessar uma região proibida classicamente, que corresponde à barreira do potencial.

Uma forma de calcular a amplitude da transição entre o mínimo metaestável e o estável é usando o método WKB da mecânica quântica. Este método consiste em obter uma solução aproximada para a equação de Schrodinger independente do tempo em uma dimensão.

Considerando uma partícula de massa unitária em $1+1$ dimensão, sujeita a um potencial $V(x)$, podemos escrever a equação de Schrodinger para a mesma da forma [54]

$$
\frac{d^{2} \psi}{d x^{2}}=\frac{2(V-E)}{\hbar^{2}} \psi
$$

Se $V(x)$ for constante temos uma solução de onda plana,

$$
\psi \propto e^{+{ }^{+} i k x}
$$

onde $k=\frac{\sqrt{2(E-V)}}{\hbar}$.

Na região classicamente proibida o momento se torna imaginário, e neste caso ao invés de uma onda plana obtemos como solução uma função de onda que decresce exponencialmente, 


$$
\psi \propto e^{-k x}
$$

onde $k=\frac{\sqrt{2(V-E)}}{\hbar}$.

Se $V(x)$, ao invés de ser constante, variar muito lentamente em relação ao comprimento de onda $\lambda=\frac{1}{k}$, então sobre uma região contendo vários comprimentos de onda o potencial é praticamente constante. Isto quer dizer que o comprimento de onda é muito pequeno em comparação aos parâmentros do potencial da partícula, ou seja,

$$
\lambda=\frac{1}{k}=\frac{\hbar}{\sqrt{2(V(x)-E)}} \rightarrow \text { muito pequeno. }
$$

Isto corresponde a termos muito pequenos em relação aos parâmentros da nossa teoria. Este limite formal em que $\hbar$ é muito pequeno é chamado limite semiclássico.

Considerando então $V(x) \sim$ cte podemos derivar a expressão (76) obtida para $\psi$. Assim chegamos à equação

$$
\frac{d \psi}{d x}={ }_{-}^{+} \frac{\sqrt{2(V(x)-E)}}{\hbar} \psi .
$$

Resolvendo para $\psi$, obtemos para a partícula que tunela para a direita

$$
\psi \propto e^{-\frac{1}{\hbar} \int \sqrt{2(V(x)-E)} d x},
$$

de modo que a amplitude do processo é dada por

$$
A \propto e^{-\frac{2}{\hbar} \int_{a}^{b} \sqrt{2(V(x)-E)} d x},
$$

onde $a$ e $b$ são os pontos de ínicio e fim da trajetória do tunelamento.

Se derivarmos a expressão (78) obtemos

$$
\frac{d^{2} \psi}{d x^{2}}=\frac{\sqrt{2(V(x)-E)}}{\hbar} \psi^{\prime}+\frac{V^{\prime}(x) \psi}{\hbar \sqrt{2(V(x)-E)}},
$$

que é igual a

$$
\frac{d^{2} \psi}{d x^{2}}=\frac{2(V(x)-E)}{\hbar^{2}} \psi+\frac{V^{\prime}(x) \psi}{\hbar \sqrt{2(V(x)-E)}} .
$$

No limite formal em que $\hbar \rightarrow 0$ podemos desprezar o segundo termo do lado direito da equação, e assim obtemos a equação de Schrodinger original . 
Devemos lembrar que, enquanto não estivermos lidando com a gravitação, podemos ajustar o potencial $V(x)$ transladando o gráfico verticalmente, sem alterar as predições da teoria. Assim podemos considerar o caso em que a energia potencial é igual à cinética. Podemos escrever o expoente da amplitude como

$\int_{a}^{b} \sqrt{2(V(x)-E)} d x=\int_{a}^{b} i p d x=\int_{a}^{b} i \frac{d x}{d t} d x=i \int_{a}^{b} p \dot{x} d t=-i \int_{a}^{b} L_{m} d t=-i S_{m}$

onde $L_{m}$ é a Lagrangeana no espaço de Minkowski e $S_{m}$ é a ação correspondente.

As amplitudes podem ser escritas alternativamente na formulação de integrais de caminho na forma

$$
A=<x=b\left|e^{i H t / \hbar}\right| x=a>=\mathcal{N} \int d(x(t)) e^{i S_{m}(x(t)) / \hbar},
$$

em que $\mathcal{N}$ é o fator de normalização.

É possível também trabalhar o cálculo do tunelamento através de uma outra abordagem. Se fizermos uma rotação de Wick $t \rightarrow i \tau$, do tempo real para o tempo imaginário, e passarmos para o espaço euclideano, a energia cinética da partícula passará a ser negativa. Neste caso as regiões classicamente proibidas se tornarão acessíveis [55].

Como a energia de uma partícula é dada por

$$
E=\frac{\dot{x}^{2}}{2}+V(x)
$$

classicamente uma partícula não pode atravessar uma região em que a energia potencial seja maior que a energia total da partícula, pois nestes casos a sua energia cinética seria negativa, o que é classicamente proibido. No entanto ao fazermos $t$ imaginário a energia cinética da partícula passa a ser negativa e então estas regiões em que $E<V$ podem se tornar acessíveis. Podemos então, efetuar os cálculos no espaço euclideano, para encontrarmos a taxa de decaimento que queremos.

Calcular a probabilidade de transição para uma partícula tunelar através de uma região classicamente proibida pela integral de caminho no espaço de Minkowski corresponde a calcular a amplitude para a partícula tunelar através de uma região classicamente permitida no espaço euclideano. Neste espaço, a nossa integral (83) fica: 


$$
-\int_{\tau_{a}}^{\tau_{b}} p \dot{x} d \tau=\int_{\tau_{a}}^{\tau_{b}} L_{e} d \tau=S_{e}
$$

onde $L_{e}$ é a Lagrangeana no espaço euclideano e $S_{e}$ é a ação no mesmo espaço. Assim, a amplitude será dada, na formulação de integrais de caminho, por

$$
A=<x=b\left|e^{-H \tau / \hbar}\right| x=a>=\mathcal{N} \int d(x(\tau)) e^{-S_{e}(x(\tau)) / \hbar} .
$$

Podemos expandir esta matriz da forma

$$
A=<x=b\left|e^{-H \tau / \hbar}\right| x=a>=\sum_{n}<b|n><n| a>e^{-E_{n} \tau / \hbar} .
$$

A largura de decaimento de uma partícula em um estado metaestável de um potencial como o descrito na figura 5.1 por exemplo, corresponde à parte imaginária da energia referente a este estado, que é a parte que tende a decair. Um estado metaestável tem uma energia que tem partes real e imaginária,

$$
E_{0}=\varepsilon+i \Gamma / 2,
$$

onde $\Gamma$ corresponde à largura de decaimento da partícula. Para encontrar a expressão desta largura devemos então encontrar a expressão da energia correspondente a este estado. No caso de uma energia puramente real, quando o estado é estável, é possível encontrar a expressão desta energia a partir da amplitude transição obtida acima. Começaremos então pelo caso estável com energia real. Supomos que a partícula tunele a partir do repouso. Para encontrarmos $E_{0}$ fazemos então $a=b$. Quando $\tau \rightarrow \infty$ o sistema tende a ir para os estados de mais baixa energia, portanto,

$$
A=<a\left|e^{-H \tau / \hbar}\right| a>\underset{\tau \rightarrow \infty}{\approx}|<a| 0>\left.\right|^{2} e^{-E_{0} \tau / \hbar}+\ldots
$$

A amplitude $A$ também pode ser expressa pela integral de caminho (87) com as condições de contorno

$$
x(-T / 2)=x(T / 2)=a,
$$

onde $T$ é o intervalo de tempo, que tenderá a infinito. Escolhemos o intervalo de $-T / 2$ a $T / 2$ apenas por conveniência. 
Esta integral de caminho corresponde a uma soma sobre todos os caminhos que têm estas condições inicial e final. No entanto, como o integrando é $e^{-S_{e}(x(\tau)) / \hbar}$ vemos que a maior contribuição virá dos caminhos que possuem menor ação, e como sabemos, os caminhos de menor ação são os caminhos clássicos. Portanto a amplitude é dominada pela solução clássica. Então podemos expandir a ação em torno dos caminhos clássicos, ou seja, aqueles caminhos cuja ação segue a equação $\frac{\delta S}{\delta x(\tau)}=0$. Expandindo a ação em série de Taylor obtemos

$$
S_{e}(x(\tau))=S\left(x_{c}(\tau)\right)+\left.\frac{1}{2} \int d \tau^{\prime} d \tau^{\prime \prime} \frac{\delta^{2} S_{e}(x(\tau))}{\delta x\left(\tau^{\prime}\right) \delta x\left(\tau^{\prime \prime}\right)}\right|_{x(\tau)=x_{c}(\tau)} \delta x\left(\tau^{\prime}\right) \delta x\left(\tau^{\prime \prime}\right)+\ldots,
$$

onde $x_{c}$ é o valor clássico de $x$ e $\delta x(\tau)=x(\tau)-x_{c}(\tau)$. A primeira derivada é nula devido à equação de movimento da trajetória clássica. A derivada funcional é dada por

$$
\begin{gathered}
\left.\frac{\delta^{2} S_{e}(x(\tau))}{\delta x\left(\tau^{\prime}\right) \delta x\left(\tau^{\prime \prime}\right)}\right|_{x(\tau)=x_{c}(\tau)}=\left.\frac{1}{\delta x\left(\tau^{\prime}\right) \delta x\left(\tau^{\prime \prime}\right)} \delta^{2}\left\{\left(\frac{-d_{\tau} x(\tau) d_{\tau} x(\tau)}{2}\right)+V\right\}\right|_{x(\tau)=x_{c}(\tau)}, \\
\left.\frac{\delta^{2} S_{e}(x(\tau))}{\delta x\left(\tau^{\prime}\right) \delta x\left(\tau^{\prime \prime}\right)}\right|_{x(\tau)=x_{c}(\tau)}=\left\{-\frac{d^{2}}{d \tau^{\prime 2}}+V^{\prime \prime}\left(x_{c}\left(\tau^{\prime}\right)\right)\right\} \delta\left(\tau^{\prime}-\tau^{\prime \prime}\right) .
\end{gathered}
$$

Então, a expansão da ação fica sendo

$$
S_{e}(x(\tau))=S\left(x_{c}(\tau)\right)+\frac{1}{2} \int d \tau \delta x(\tau)\left\{-\frac{d^{2}}{d \tau^{2}}+V^{\prime \prime}\left(x_{c}(\tau)\right)\right\} \delta x(\tau)+\ldots
$$

Podemos expandir $\delta x(\tau)$ em termos de um conjunto completo de autofunções ortonormais $x_{n}(\tau)$ do operador $\left\{-\frac{d^{2}}{d \tau^{2}}+V^{\prime \prime}\left(x_{c}(\tau)\right)\right\}$. Deste modo

$$
\delta x(\tau)=\sum_{n=1}^{\infty} c_{n} x_{n}(\tau) .
$$

Portanto temos a equação

$$
\left\{-\frac{d^{2}}{d \tau^{2}}+V^{\prime \prime}\left(x_{c}(\tau)\right)\right\} x_{n}(\tau)=\lambda_{n} x_{n}(\tau) n=0,1,2,3, \ldots, \infty,
$$

sujeita às condições de contorno

$$
x_{n}(-T / 2)=x_{n}(T / 2)=a .
$$

As autofunções seguem as seguintes relações de completeza 


$$
\sum_{n=1}^{\infty} x_{n}(\tau) x_{n}\left(\tau^{\prime}\right)=\delta\left(\tau-\tau^{\prime}\right)
$$

e ortonormalidade

$$
\int_{-T / 2}^{T / 2} d \tau x_{n}(\tau) x_{m}(\tau)=\delta_{n m}
$$

Obtemos para a ação a seguinte expressão,

$$
S_{e}(x(\tau))=S_{e}\left(x_{c}(\tau)\right)+\frac{1}{2} \sum_{n=1}^{\infty} \lambda_{n} c_{n}^{2}+o\left(c_{n}^{3}\right) .
$$

Portanto a amplitude que queremos calcular pode ser escrita como

$$
\begin{gathered}
<a\left|e^{-T \hat{H} / \hbar}\right| a>=\mathcal{N} \int d x(\tau) e^{-\frac{1}{\hbar}\left\{S_{e}\left(x_{c}(\tau)\right)+\frac{1}{2} \sum_{n=1}^{\infty} \lambda_{n} c_{n}^{2}+o\left(c_{n}^{3}\right)\right\}} \\
=\mathcal{N} e^{-\frac{1}{\hbar} S_{e}\left(x_{c}(\tau)\right)} \int d x(\tau) e^{-\frac{1}{\hbar}\left\{\frac{1}{2} \sum_{n=1}^{\infty} \lambda_{n} c_{n}^{2}+o\left(c_{n}^{3}\right)\right\}} .
\end{gathered}
$$

Precisamos agora mudar o fator de integração da forma

$$
\mathcal{D} x(\tau) \rightarrow \prod_{n=1}^{\infty} \frac{d c_{n}}{\sqrt{2 \pi \hbar}} .
$$

O fator $\sqrt{2 \pi \hbar}$ é colocado por conveniência e pode ser absorvido na constante de normalização $\mathcal{N}$.

A equação da amplitude então se torna

$$
<a\left|e^{-T \hat{H} / \hbar}\right| a>=e^{-S_{e}\left(x_{c}(\tau)\right) / \hbar} \mathcal{N} \prod_{n=1}^{\infty} \frac{d c_{n}}{\sqrt{2 \pi \hbar}} e^{-\frac{1}{\hbar}\left(\frac{1}{2} \lambda_{n} c_{n}^{2}+o\left(c_{n}^{3}\right)\right)} .
$$

Por conveniência podemos substituir $c_{n} \rightarrow \tilde{c}_{n} \sqrt{\hbar}$, e assim obtemos

$$
\begin{aligned}
& <a\left|e^{-T \hat{H} / \hbar}\right| a>=e^{-S_{e}\left(x_{c}(\tau)\right) / \hbar} \mathcal{N} \prod_{n=1}^{\infty} \frac{d \widetilde{c}_{n}}{\sqrt{2 \pi}} e^{-\left(\frac{1}{2} \lambda_{n} \widetilde{c}_{n}^{2}+o(\hbar)\right)}, \\
& <a\left|e^{-T \hat{H} / \hbar}\right| a>=e^{-S_{e}\left(x_{c}(\tau)\right) / \hbar} \mathcal{N} \prod_{n=1}^{\infty}\left\{\frac{1}{\sqrt{\lambda_{n}}}(1+o(\hbar))\right\} .
\end{aligned}
$$

Podemos então escrever a amplitude como [56]

$$
\left.<a\left|e^{-T \hat{H} / \hbar}\right| a>=e^{-S_{e}\left(x_{c}(\tau)\right) / \hbar} \mathcal{N}\left\{\operatorname{det}\left[-\frac{d^{2}}{d \tau^{2}}+V^{\prime \prime}\left(x_{c}(\tau)\right)\right]\right\}^{-\frac{1}{2}}(1+o(\hbar))\right\} .
$$


A integral de caminho pode ser obtida no caso de uma teoria de campos de forma completamente análoga à mecânica quântica, com pequenas mudanças. Para a medida da integração, ao invés de utilizarmos coordenadas usamos campos. No caso das partículas, as variáveis eram as posições das partículas no espaço e estas posições variavam no tempo. Já no caso dos campos, as coordenadas espaciais não são mais posições de partículas, são apenas parâmetros. A variável dinâmica neste caso possui um número infinito de graus de liberdade, ao contrário do caso anterior.

Podemos supor uma forma euclideana $\mathcal{O}(4)$ invariante para o campo,

$$
\phi(\vec{x}, \tau)=\phi\left(|\vec{x}|^{2}+\tau^{2}\right)^{\frac{1}{2}}=\phi(\rho),
$$

em que $\rho$ representa as coordenadas de espaço e tempo.

Desta forma nossa ação para os campos fica

$$
S_{e}(\phi)=2 \pi^{2} \int_{0}^{\infty} d \rho \rho^{3}\left[\frac{1}{2}\left(\frac{\partial \phi}{\partial \rho}\right)^{2}+V(\phi)\right] .
$$

Supondo o caso do potencial da figura 5.1 podemos calcular a amplitude supondo desta vez a condição de contorno $\lim _{\rho \rightarrow{ }_{-}^{+}} \phi(\rho)=\phi_{+}$, em que $\phi_{+}$é o valor do campo no mínimo metaestável do potencial. Por conveniência supomos um intervalo de tempo de $-\frac{T}{2}$ a $+\frac{T}{2}$. Vemos que existem duas soluções possíveis com estas condicões de contorno: uma delas corresponde ao caso estático em que o campo permanece em $\phi_{+}$por um tempo infinito, e a outra corresponde ao caso oscilatório em que o campo interpola entre os dois pontos de mínimo do potencial, saindo de $\phi_{+}$e retornando ao mesmo ponto. Neste caso supomos a condicão de contorno $\left.\frac{d \phi}{d \rho}\right|_{\rho=0}=0$.

Podemos expandir nossa ação como no caso anterior, e obtemos que nossa amplitude pode ser expressa como

$$
<\phi_{+}\left|e^{-T \hat{H} / \hbar}\right| \phi_{+}>=e^{-S_{e}\left(\phi_{o}(\rho)\right) / \hbar} \mathcal{N}\left\{\operatorname{det}\left[-\partial_{\mu} \partial_{\mu}+V^{\prime \prime}\left(\phi_{o}(\rho)\right)\right]\right\}^{-\frac{1}{2}},
$$

onde $\left(\phi_{o}(\rho)\right)$ corresponde à solução não estática da equação de movimento do campo. O determinante normalizado acima pode ser escrito na forma

$$
\mathcal{N}\left\{\operatorname{det}\left[-\partial_{\mu} \partial_{\mu}+V^{\prime \prime}\left(\phi_{o}(\rho)\right)\right]\right\}^{-\frac{1}{2}} \rightarrow \mathcal{K}\left(\operatorname{det}\left(-\partial_{\mu} \partial_{\mu}+w^{2}\right)\right)^{-\frac{1}{2}}
$$

onde 


$$
\mathcal{K}=\frac{\left\{\operatorname{det}\left[-\partial_{\mu} \partial_{\mu}+V^{\prime \prime}\left(\phi_{o}(\rho)\right]\right\}^{-\frac{1}{2}}\right.}{\left(\operatorname{det}\left(-\partial_{\mu} \partial_{\mu}+w^{2}\right)\right)^{-\frac{1}{2}}},
$$

em que $w^{2}=V^{\prime \prime}\left(\phi_{+}\right)$, correspondente à solução estática.

A equação de movimento clássica do campo $\phi$, no espaço euclideano, que possui um potencial $V(\phi)$, é obtida minimizando-se a ação,

$$
\frac{\delta S e(\phi(x))}{\delta \phi}=\partial_{\mu} \partial_{\mu} \phi(\rho)-V^{\prime}(\phi(\rho))=0 .
$$

Daí obtemos a equação de movimento do campo escalar

$$
\frac{d^{2} \phi}{d \rho^{2}}+\frac{3}{\rho} \frac{d \phi}{d \rho}-V^{\prime}(\phi)=0 .
$$

Ver Apêndice B. (A partir daqui o símbolo' sempre indicará uma derivada com relação ao campo, a menos que seja especificado de outra forma.)

A equação acima pode ser interpretada de forma análoga à equação para uma partícula na posição $\phi$ movendo-se no tempo $\rho$, sujeita a uma força igual á $-V^{\prime}$ e á uma força com forma análoga á uma força de atrito com um coeficiente dependendo de $\rho$. Uma solução desta equação com as condições de contorno que queremos corresponde a uma oscilação entre os dois máximos do potencial $-V$. O segundo termo nesta equação de movimento tem seu sinal revertido no percurso de volta, e apesar de não ser de fato um atrito, mas apenas um termo dissipativo, chamaremos deste modo devido à sua forma análoga.

O potencial invertido desta partícula está representado na figura (6.1) [55]

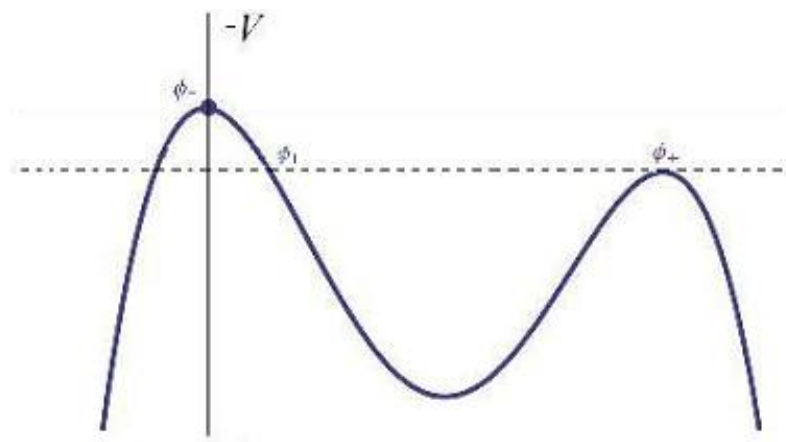

Figura 6.1: O potencial invertido. 
Podemos provar que existe uma solução em que a partícula sai do ponto $\phi_{-}$e chega ao ponto $\phi_{+}$, o que corresponde à metade do percurso que queremos.

Supomos a partícula inicialmente em um ponto $\phi_{i}$ muito próximo de $\phi_{-}$, com velocidade inicial igual á zero, no instante inicial $\tau=0 . \phi_{i}>\phi_{1}$, sendo $\phi_{1}$ o ponto em que $V=0$, como mostra a figura. A partícula no seu movimento sairá inicialmente da posição próxima ao máximo com velocidade muito pequena, pois como o coeficiente de atrito é $\frac{3}{\rho}$ e como $\rho$ é inicialmente muito pequeno, o atrito é inicialmente muito grande. Então o tempo para que a partícula chegue próximo de $\phi_{1}$ pode ser tão longo que o coeficiente de atrito passa então a ser desprezível, e a partir daí o sistema passa a se comportar como um sistema conservativo. Existe certamente uma posição inicial $\phi_{i}$ tal que a partícula chegue exatamente em $\phi_{+}$com velocidade zero. Como a partícula fica um longo tempo próxima à posição inicial, e então rapidamente cruza o potencial no instante próximo de $T=0$, chamamos esta solução de instanton [55]. Revertendo o movimento temos outra solução que inicia em $\phi_{+}$e chega em $\phi_{-}$. Esta solução tem mesma ação que a anterior e é chamada anti-instanton.

Como estamos tratando do caso em que o tempo é levado para infinito, temos então uma simetria de translação temporal, ou seja, também haverá outras soluções com praticamente a mesma ação (a ação da solução clássica), que são da mesma forma que a solução descrita acima, porém estas soluções são tais que o momento em que a partícula cruza o potencial ocorre em um instante diferente. Como estamos lidando com um intervalo de tempo $\tau=T$ a degenerescência da solução que segue a equação de movimento clássica é dada por $T$. Como nestes casos $\delta S \approx 0$, a translação temporal representada por $\phi$, satisfaz

$$
\left[\partial_{\mu} \partial_{\mu}-V^{\prime \prime}\left(\phi_{o}(\rho)\right)\right] \dot{\phi}=0 .
$$

Isto mostra que temos um modo nulo no determinante. A melhor forma de lidar com isto é excluindo este modo nulo da expressão do determinante e integrando a solução sob as posições (no espaço-tempo) da oscilação. Ao integrar sobre as posições no espaço-tempo nossa solução ficará então multiplicada por T.V (tempo vezes volume) e por um fator de normalização igual a $\left(\sqrt{\frac{S_{e}\left(\phi_{o}(\rho)\right)}{2 \pi \hbar}}\right)^{D}$, onde $D$ é a dimensão do espaço-tempo [57]. Este fator vem do jacobiano da transformação da variável de integração. Precisamos 
lembrar que o limite exato em que o tempo é infinito zera a amplitude que queremos calcular, então o que calculamos na verdade é um limite para $T$ muito grande, tendendo a infinito, e por isso o modo nulo não é exatamente zero, mas tende a zero neste limite. Nossa amplitude pode então ser escrita como [57]

$$
<\phi_{+}\left|e^{-T \hat{H} / \hbar}\right| \phi_{+}>=\frac{\left(S_{e}\left(\phi_{o}(\rho)\right)\right)^{2}}{(2 \pi \hbar)^{2}} T V e^{-S_{e}\left(\phi_{o}(\rho)\right) / \hbar}\left\{\operatorname{det}\left(-\partial_{\mu} \partial_{\mu}+w^{2}\right)\right\}^{-\frac{1}{2}}\left\{\left(\frac{\operatorname{det}^{\prime}\left(-\partial_{\mu} \partial_{\mu}+V^{\prime \prime}\left(\phi_{o}(\rho)\right)\right.}{\operatorname{det}\left(-\partial_{\mu} \partial_{\mu}+w^{2}\right)}\right)^{-\frac{1}{2}}\right\} .
$$

O símbolo' no determinante indica que o modo zero foi excluído.

A solução de uma única oscilação (um instanton e um anti-instanton) não é a única. Existem outros pontos críticos aproximados que contribuem significativamente para a integral de caminho. Se tivermos por exemplo, ao invés de uma ida e volta, várias idas e voltas, cada uma bem separada dentro do intervalo de tempo $\mathrm{T}$, esta também será uma solução que minimiza a ação. Teremos neste caso $n$ pares de instantons - anti-instantons com posições arbitrárias [55]. A ação para $n$ oscilações será aproximadamente $n S_{e}\left(\phi_{o}(\rho)\right)$, e a degenerescência desta solução é $\frac{T^{n}}{n !}$, devido à invariância de translação temporal de cada uma. Isto ocorre para $T \rightarrow \infty$, mas para $T$ muito grande é uma boa aproximação.

O determinante das $n$ oscilações pode ser obtido fazendo-se a substituição

$$
\left\{\left(\frac{\operatorname{det}^{\prime}\left(-\partial_{\mu} \partial_{\mu}+V^{\prime \prime}\left(\phi_{O}(\rho)\right)\right.}{\operatorname{det}\left(-\partial_{\mu} \partial_{\mu}+w^{2}\right)}\right)^{-\frac{1}{2}}\right\} \rightarrow\left\{\left(\frac{\operatorname{det}^{\prime}\left(-\partial_{\mu} \partial_{\mu}+V^{\prime \prime}\left(\phi_{O}(\rho)\right)\right.}{\operatorname{det}\left(-\partial_{\mu} \partial_{\mu}+w^{2}\right)}\right)^{-\frac{n}{2}}\right\} .
$$

Somando para todos os possíveis valores de $n$ obtemos a amplitude

$<\phi_{+}\left|e^{-T \hat{H} / \hbar}\right| \phi_{+}>=\left\{\operatorname{det}\left[-\partial_{\mu} \partial_{\mu}+w^{2}\right]\right\}^{-\frac{1}{2}} \sum_{n=0}^{\infty} \frac{\left(S_{e}\left(\phi_{O}(\rho)\right)\right)^{2 n}}{(2 \pi \hbar)^{2 n}}\left(\frac{(T V)^{n}}{n !}\right) e^{-n S_{e}\left(\phi_{O}(\rho)\right) / \hbar}\left\{\left(\frac{\operatorname{det}^{\prime}\left(-\partial_{\mu} \partial_{\mu}+V^{\prime \prime}\left(\phi_{o}(\rho)\right)\right.}{\operatorname{det}\left(-\partial_{\mu} \partial_{\mu}+w^{2}\right)}\right)^{-\frac{n}{2}}\right\}$.

Esta expressão corresponde a

$$
<\phi_{+}\left|e^{-T \hat{H} / \hbar}\right| \phi_{+}>=\left(\operatorname{det}\left[-\partial_{\mu} \partial_{\mu}+w^{2}\right]\right)^{-\frac{1}{2}} \exp \left[\frac{\left(S_{e}\left(\phi_{o}(\rho)\right)\right)^{2}}{(2 \pi \hbar)^{2}} T V e^{-S_{e}\left(\phi_{O}(\rho)\right) / \hbar}\left\{\left(\frac{\operatorname{det}^{\prime}\left(-\partial_{\mu} \partial_{\mu}+V^{\prime \prime}\left(\phi_{o}(\rho)\right)\right.}{\operatorname{det}\left(-\partial_{\mu} \partial_{\mu}+w^{2}\right)}\right)^{-\frac{1}{2}}\right\}\right] .
$$

Analogamente ao caso que vimos para a mecânica quântica temos que

$$
A=<\phi_{+}\left|e^{-H \tau / \hbar}\right| \phi_{+}>\underset{\tau \rightarrow \infty}{\approx}\left|<\phi_{+}\right| 0>\left.\right|^{2} e^{-E_{0} \tau / \hbar}+\ldots
$$


Vemos que a energia $E_{0}$ corresponde ao termo no exponte multiplicado por $\frac{\hbar}{\tau}$. Temos que

$$
\left\{\operatorname{det}\left[-\partial_{\mu} \partial_{\mu}+w^{2}\right]\right\}^{-\frac{1}{2}}=\left(\frac{w}{\pi \hbar}\right)^{\frac{1}{2}} e^{-\frac{w \tau}{2}},
$$

pois a expressão no determinante corresponde à expressão de um oscilador harmônico livre. Deste modo, podemos encontrar $E_{0}$,

$$
E_{0}=\frac{\hbar w}{2}+\hbar \frac{\left(S_{e}\left(\phi_{o}(\rho)\right)\right)^{2}}{(2 \pi \hbar)^{2}} V e^{-S_{e}\left(\phi_{o}(\rho)\right) / \hbar}\left\{\left(\frac{\operatorname{det}^{\prime}\left(-\partial_{\mu} \partial_{\mu}+V^{\prime \prime}\left(\phi_{o}(\rho)\right)\right.}{\operatorname{det}\left(-\partial_{\mu} \partial_{\mu}+w^{2}\right)}\right)^{-\frac{1}{2}}\right\} .
$$

No entanto esta ainda não é a largura de decaimento que procuramos, pois a mesma corresponde à parte imaginária da energia, e a energia acima é real. Ocorre, porém, que o autovetor de modo zero dado pela função $\phi$, tem um sinal negativo na ida e positivo na volta, ou seja, a função possui um zero. No entanto sabe-se que o estado de menor autovalor de um sistema não possui um zero. Isto indica que existe um autovalor menor que o nulo, ou seja, um autovalor negativo [56].

Como o determinante na equação acima é dado pela raiz do produto dos autovalores, então o determinante será imaginário. O determinante imaginário dará a contribuição imaginária que precisamos para a energia correspondente ao estado metaestável. Apenas a parte imaginária da energia contribuirá para a largura de decaimento. Portanto a energia imaginária que buscamos vem apenas do segundo termo do lado direito da equacão (123), pois o primeiro é real. Então esperaríamos que [56]

$$
\mathcal{I} m E_{0}=\frac{\Gamma}{2}=\hbar \frac{\left(S_{e}\left(\phi_{o}(\rho)\right)\right)^{2}}{(2 \pi \hbar)^{2}} V\left\{\left(\frac{\operatorname{det}\left(-\partial_{\mu} \partial_{\mu}+V^{\prime \prime}\left(\phi_{o}(\rho)\right)\right.}{\operatorname{det}\left(-\partial_{\mu} \partial_{\mu}+w^{2}\right)}\right)^{-\frac{1}{2}}\right\} e^{-S_{e}\left(\phi_{o}(\rho)\right) / \hbar},
$$

se

$$
A=<\phi_{+}\left|e^{-H T / \hbar}\right| \phi_{+}>=<\phi_{+}\left|\varepsilon+i \frac{\Gamma}{2}><\varepsilon+i \frac{\Gamma}{2}\right| \phi_{+}>e^{-\left(\varepsilon+i \frac{\Gamma}{2}\right) T / \hbar} .
$$

Mas esta expressão não é bem definida. Não há nenhum estado da Hamiltoniana que corresponda a um estado metaestável. A Hamiltoniana é um operador hermiteano com todos os autovalores reais. Podemos obter a energia do estado metaestável apenas através de continuação analítica. A integral de caminho que calculamos para a amplitude do tunelamento na realidade 
não é bem definida para o caso de um potencial com um mínimo metaestável como o da figura 5.1.

Como não podemos calcular diretamente a energia do estado metaestável supomos inicialmente um outro potencial, com um mínimo estável, que é função de um parâmetro $\alpha$. Para potenciais deste tipo em que o mínimo é estável não há nenhum problema com a integral de caminho que calculamos e o mínimo estável possui uma energia bem definida. Agora supomos que podemos alterar este potencial fazendo uma continuação analítica neste parâmetro $\alpha$ de tal forma que obtemos a partir dele o potencial que queremos, com o mínimo metaestável. A sequência de potenciais citada acima está representada na figura abaixo [55].

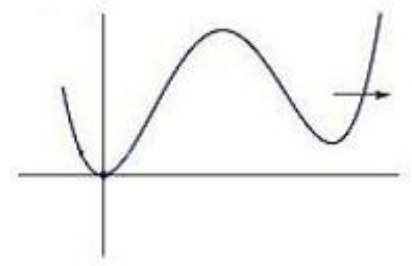

Figura 6.2 a: $\alpha=0$.

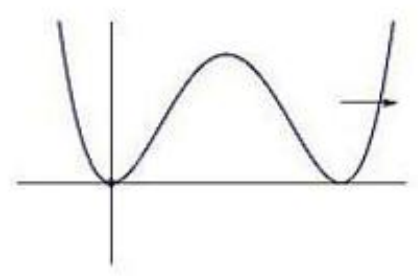

Figura 6.2 b: $\alpha$ crítico.

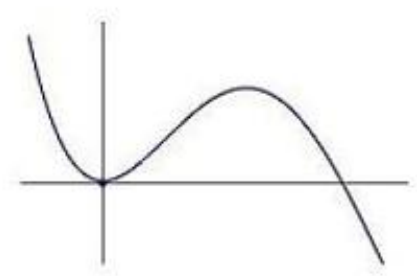

Figura 6.2 c: $\alpha=1$.

Quando fazemos a continuação analítica neste parâmetro $\alpha$ esperamos que a energia ganhe uma parte imaginária, ou seja, recuperamos nosso potencial com mínimo metaestável. O processo de continuação analítica em $\alpha$ envolve uma série de cálculos cujos detalhes não nos interessam aqui. Porém, pode-se mostrar que ao final é obtida a seguinte expressão para a largura de decaimento por unidade de volume [53],

$$
\frac{\Gamma}{V}=\hbar \frac{\left(S\left(\phi_{o}(\rho)\right)\right)^{2}}{(2 \pi \hbar)^{2}} e^{-S_{e}\left(\phi_{o}(\rho)\right) / \hbar} \frac{1}{\sqrt{ } \lambda_{-}}\left\{\left(\frac{\operatorname{det}^{\prime}\left(-\partial_{\mu} \partial_{\mu}+V^{\prime \prime}\left(\phi_{o}(\rho)\right)\right.}{\operatorname{det}\left(-\partial_{\mu} \partial_{\mu}+w^{2}\right)}\right)^{-\frac{1}{2}}\right\} .
$$

Vemos que nossa expressão original fora alterada apenas por um fator de $\frac{1}{2}$ que vem do processo de continuação analítica, e agora o símbolo ' no determinante representa que foram excluídos os modos nulo e negativo, sendo que o último foi considerado no fator $\frac{1}{\sqrt{ } \lambda_{-}}$.

Durante os cálculos da ação consideramos uma energia total nula, o que equivale a termos transladado o potencial de modo a termos $V=0$ no mínimo metaestável. O que calculamos na verdade foi então uma diferença entre a 
ação da solução oscilatória e a ação da solução estática no vácuo metaestável. Deste modo, é mais correto reescrevermos nossa equação da largura de decaimento por unidade de volume na forma [53]

$$
\frac{\Gamma}{V}=\frac{\hbar S_{e}\left(\phi_{0}(\rho)\right)^{2}}{(2 \pi \hbar)^{2}} e^{-\left(\frac{S_{e}}{\hbar}-\frac{S_{\Lambda}}{\hbar}\right)}\left(\frac{\operatorname{det}^{\prime}\left(-\partial_{\mu} \partial_{\mu}+V^{\prime \prime}\left(\phi_{0}(\rho)\right)\right.}{\operatorname{det}\left(-\partial_{\mu} \partial_{\mu}+V^{\prime \prime}\left(\phi_{+}\right)\right)}\right)^{-\frac{1}{2}},
$$

onde $S_{\Lambda}$ corresponde à ação da solução estacionária no falso vácuo, e 'indica a exclusão do autovalor nulo do determinante. Consideramos a dimensão do espaço-tempo igual a quatro.

Estamos trabalhando no sistema de unidades $\hbar=c=1$. Nestas unidades a expressão da taxa de decaimento por unidade de volume terá dimensão de massa $^{4}$, que equivale ao inverso da unidade de volume pelo inverso do tempo. O inverso da expressão da taxa de decaimento por unidade de volume de uma partícula nos fornece o tempo de decaimento desta partícula vezes o volume. No caso em que estamos trabalhando temos uma simetria espaço-temporal euclideana. Neste caso obtemos o tempo de decaimento calculando a raiz quarta da expressão do inverso da taxa de decaimento por volume obtida acima. Ou seja,

$$
t_{\text {decaimento }}=\left(\frac{1}{\frac{\Gamma}{V}}\right)^{\frac{1}{4}} .
$$

No capítulo seguinte calcularemos o tempo de decaimento de uma partícula de energia escura representada pelo modelo descrito no capítulo 5. Para isto usaremos a expressão da taxa de decaimento obtida acima. 


\section{7- O Decaimento da Energia Escura}

Vimos que a taxa de decaimento (por unidade de tempo e volume) de uma partícula descrita por um potencial $V(\phi)$ é dada pela expressão

$$
\frac{\Gamma}{V}=\frac{\hbar S_{e}\left(\phi_{0}(\rho)\right)^{2}}{(2 \pi \hbar)^{2}} e^{-\left(\frac{S_{e}}{\hbar}-\frac{S_{\Lambda}}{\hbar}\right)}\left(\frac{\operatorname{det}^{\prime}\left(-\partial_{\mu} \partial_{\mu}+V^{\prime \prime}\left(\phi_{0}(\rho)\right)\right.}{\operatorname{det}\left(-\partial_{\mu} \partial_{\mu}+V^{\prime \prime}\left(\phi_{+}\right)\right)}\right)^{-\frac{1}{2}} .
$$

Como vimos, $\phi_{0}(\rho)$ corresponde, na analogia ao caso de partículas, ao caminho clássico, no espaço euclideano, que atravessa o potencial $-V(\phi) \mathrm{com}$ as condições de contorno $\phi_{\text {inicial }}=\phi_{\text {final }}=\phi_{\text {minmetaestável }}$, que corresponde, na nossa analogia, à oscilação de uma partícula, entre os dois máximos do potencial $-V(\phi)$. $\rho$ representa as coordenadas espaciais e temporais.

Podemos agora calcular a taxa de decaimento da partícula de energia escura descrita pelo modelo proposto no capítulo 5, em que a mesma é descrita por um potencial supersimétrico de Wess-Zumino, $U=\left|2 m \varphi-3 \lambda \varphi^{2}\right|^{2}$, somado a um termo de quebra de supersimetria. O potencial de Wess-Zumino tem dois mínimos, o primeiro localizado em $\varphi=0$ e o segundo em $\varphi=\frac{2 m}{3 \lambda}$. Á ele adicionaremos um termo de quebra de supersimetria do tipo potência em $\varphi$. A forma específica deste termo não nos interessa por enquanto, apenas queremos que no ponto de mínimo metaestável, correspondente ao segundo mínimo do potencial, o valor do potencial seja igual a $\epsilon=10^{-47} \mathrm{GeV}^{4}$, que corresponde à densidade de energia da constante cosmológica,

$$
V\left(\varphi_{\text {mínmetaestável }}\right)=10^{-47} \mathrm{GeV}^{4},
$$

sendo que

$$
V(\varphi)=U(\varphi)+Q(\varphi)
$$


onde $Q(\varphi)$ é o termo de quebra de supersimetria.

Podemos calcular o tempo de decaimento do mínimo metaestável para o mínimo estável em função da massa da partícula de energia escura, o que corresponde a um possível decaimento de energia escura em matéria escura. Sabemos que se considerarmos o potencial de Wess-Zumino com seu máximo da altura do mínimo metaestável teríamos um decaimento imediato. Então, podemos concluir que para determinados valores dos parâmetros de nossa teoria, a barreira do potencial terá a altura exata necessária para um decaimento cujo tempo corresponde ao tempo de vida do universo, o que é requerido devido ao longo tempo de vida da partícula de energia escura.

Usaremos o potencial supersimétrico de Wess-Zumino como motivação e consideraremos apenas a parte real do campo bosônico, o que, como vimos, corresponde a uma teoria de gravitação $f(R)$.

O cálculo da taxa de decaimento é um cálculo complicado e que geralmente não possui uma solução analítica. Porém, em alguns casos, quando a diferença de energia entre os dois mínimos do potencial for muito pequena, podemos usar uma aproximação que nos permite obter uma solução analítica para o problema. Esta aproximação é chamada aproximação da parede fina (thin wall limit), por motivos que serão esclarecidos posteriormente. Nesta aproximação, como a diferença de energia entre os dois mínimos, dada pelo parâmetro $\epsilon$, é relativamente pequena, podemos efetuar nossos cálculos perturbativamente em $\epsilon$.

Como queremos calcular a taxa de decaimento devido ao tunelamento da partícula para o mínimo estável, só estamos interessados nos casos em que a barreira do nosso potencial é maior que o valor do potencial no mínimo metaestável. Quando o mínimo do potencial fica da mesma altura do máximo, o valor do potencial no ponto de máximo é igual ao valor da constante cosmológica. Assim temos a seguinte condição para que o potencial possua uma barreira,

$$
V_{\text {máx }}>10^{-47} \mathrm{GeV}^{4}=\text { constante cosmológica. }
$$

No ponto de máximo do potencial supersimétrico o valor do campo é $\varphi_{\text {máxss }}=\frac{m}{3 \lambda}$. Ao adicionarmos o termo de quebra de supersimetria este valor do campo no ponto de máximo é alterado para $\varphi_{\text {máx }}=\frac{m}{3 \lambda}+\delta \varphi$. Podemos aproximar o valor de $V\left(\varphi_{\text {máx }}\right)$ expandindo-o em torno do valor de $\varphi$ correspondente ao ponto de máximo do potencial supersimétrico: $\varphi_{\text {máxss }}=\frac{m}{3 \lambda}$.

Lembramos que o potencial pode ser escrito como 


$$
V\left(\varphi_{\operatorname{máx}}\right)=U\left(\varphi_{\operatorname{máx}}\right)+Q\left(\varphi_{\operatorname{máx}}\right) .
$$

Podemos expandir a parte supersimétrica em torno de $\varphi_{\text {máxss }}=\frac{m}{3 \lambda}$ da forma

$$
U\left(\varphi_{\text {máx }}\right)=U\left(\varphi_{\text {máxss }}\right)+U^{\prime}\left(\varphi_{\text {máxss }}\right) \delta \varphi+U^{\prime \prime}\left(\varphi_{\text {máxss }}\right) \delta \varphi^{2} .
$$

Mantendo apenas os termos até primeira ordem e lembrando que $U^{\prime}\left(\varphi_{\text {máxss }}\right)=$ 0 , obtemos para o termo supersimétrico:

$$
U\left(\varphi_{\text {máx }}\right) \sim U\left(\varphi_{\text {máxss }}\right)
$$

Já o termo de quebra de supersimetria, sendo uma potência de $\varphi$, obedecerá no ponto de máximo à desigualdade $\mathrm{Q}(\varphi)<10^{-47} \mathrm{GeV}^{4}$, pois o valor de $\varphi$ no ponto de máximo é menor que o valor de $\varphi$ no ponto de mínimo metaestável onde $V(\varphi)=10^{-47} \mathrm{GeV}^{4}$. Então calcularemos primeiro a condição $V_{\text {máx }}>10^{-47} \mathrm{GeV}^{4}$ levando em conta apenas a parte supersimétrica, e depois acrescentaremos a parte do termo de quebra. Assim, temos a condição

$$
U\left(\varphi_{\text {máxss }}\right)>10^{-47} \mathrm{GeV}^{4} .
$$

Substituindo o valor do campo $\varphi_{\text {máxss }}=\frac{m}{3 \lambda}$ na expressão do potencial $\mathrm{U}(\varphi)$, a nossa condição fica

$$
9 \lambda^{2}\left(\frac{m}{3 \lambda}\right)^{4}+4 m^{2}\left(\frac{m}{3 \lambda}\right)^{2}-12 m \lambda\left(\frac{m}{3 \lambda}\right)^{3}>10^{-47} \mathrm{GeV}^{4} .
$$

Obtemos assim,

$$
\frac{m^{4}}{9 \lambda^{2}}>10^{-47}
$$

Para $\lambda=10^{-2}$ obtemos para a massa a condição

$$
\begin{aligned}
& m^{4}>\sim 10^{-51} \mathrm{GeV}, \\
& m>\sim 10^{-13} \mathrm{GeV} .
\end{aligned}
$$

Se na condição que obtivemos, $\frac{m^{4}}{9 \lambda^{2}}>10^{-47}$, acrescentássemos ao termo do lado esquerdo a contribuição do termo de quebra de supersimetria, que 
seria uma fração de $10^{-47}$, a ordem de grandeza do nosso resultado não seria alterada, portanto podemos considerar o resultado acima como uma boa aproximação.

Vimos que a equação de movimento do campo escalar é dada por

$$
\frac{\partial^{2} \varphi}{\partial \rho^{2}}+\frac{3}{\rho} \cdot \frac{\partial \varphi}{\partial \rho}-V^{\prime}(\varphi)=0
$$

com a condição de contorno $\lim _{\rho \rightarrow \rightarrow_{-}^{+}} \varphi(\rho)=\varphi_{+}$. Na equação acima' indica derivada com relação a $\varphi$.

Observando a simetria do problema é fácil ver que o decaimento ocorre através da formação de bolhas de vácuo verdadeiro cercadas pelo falso vácuo externo. $\frac{\partial \varphi}{\partial \rho}$ é diferente de zero apenas na parede da bolha, pois o campo está em repouso em $\varphi_{-}$na região interna da bolha e em $\varphi_{+}$na região externa. Se a parede da bolha é fina podemos considerar $\rho=R$ na parede (onde $R$ é o raio da bolha). Quando $R$ é muito grande, como ocorre no caso de pequenas diferenças de energia entre os dois mínimos, podemos desprezar o termo de atrito. Esta é a chamada aproximação de parede fina. Nestes casos podemos calcular a ação perturbativamente em $\epsilon$.

Sabemos que o termo exponencial da fórmula da taxa de decaimento é o termo dominante dentro da validade do limite semiclássico. Calcularemos primeiro este termo. Para isto é preciso obter a ação euclideana da solução clássica. Usando a aproximação de parede fina vamos calcular a ação de forma perturbativa em $\epsilon$. Nesta aproximação $\varphi$ é dado por

$$
\begin{gathered}
\varphi=0, \text { quando } 0<\rho \ll R, \\
\varphi=\tilde{\varphi}, \text { quando } \rho \approx R, \\
\varphi=2 m / 3 \lambda, \text { quando } \rho \gg R .
\end{gathered}
$$

O cálculo da ação pode ser separado em três regiões: fora da bolha, na parede, e dentro da bolha:

$$
S_{e}=2 \pi^{2} \int_{0}^{R-\Delta} d \rho \rho^{3}(-\epsilon)+2 \pi^{2} \int_{R-\Delta}^{R+\Delta} d \rho \rho^{3}\left(\frac{1}{2}\left(\frac{d \tilde{\varphi}}{d \rho}\right)^{2}+U\right)+2 \pi^{2} \int_{R+\Delta}^{\infty} d \rho \rho^{3}(0)
$$


Dentro e fora da bolha a energia cinética é nula pois em nossa solução o campo está, nestes casos, parado nos pontos estacionários. Devemos lembrar que calculamos a ação como sendo a diferença entre a ação da solução oscilatória de $\varphi$ e a ação da solução estacionária no falso vácuo $(V=\epsilon)$, ou seja $S_{e}=S_{o s c}-S_{\Lambda}$, por isto o interior da bolha tem $U=-\epsilon$ ao invés de $U=0$, e no exterior vice-versa. No integrando do segundo termo usamos o potencial supersimétrico $U(\varphi)$ ao invés de $V(\varphi)$, lembrando que este termo também corresponde à ação da solução oscilatória menos a ação da solução estacionária no falso vácuo, e portanto ele corresponde a

$$
2 \pi^{2} \int_{R-\Delta}^{R+\Delta} d \rho \rho^{3}\left(\frac{1}{2}\left(\frac{d \tilde{\varphi}}{d \rho}\right)^{2}+V\right)-2 \pi^{2} \int_{R-\Delta}^{R+\Delta} d \rho \rho^{3} \epsilon .
$$

Para simplificar nossos cálculos, ao invés de diminuirmos o valor $\epsilon$ do potencial $V$ diminuímos o termo de quebra de supersimetria $Q(\varphi)$, que é menor que $\epsilon$ em todos os pontos cujo valor do campo é menor que seu valor esperado de vácuo. Neste caso foi acrescentado no cálculo um erro certamente menor que

$$
2 \pi^{2} \int_{R-\Delta}^{R+\Delta} d \rho \rho^{3} \epsilon
$$

que, no limite de parede fina, é bem menor que o primeiro termo na expressão da ação, e portanto não há problemas em fazer esta aproximação.

Após integrar, obtemos a ação

$$
\begin{gathered}
S_{e} \approx-2 \pi^{2} \epsilon \frac{R^{4}}{4}+2 \pi^{2} R^{3} \int_{R-\Delta}^{R+\Delta} d \rho\left(\frac{1}{2}\left(\frac{d \tilde{\varphi}}{d \rho}\right)^{2}+U\right)+0 \\
=-\frac{1}{2} \pi^{2} R^{4} \epsilon+2 \pi^{2} R^{3} S_{1},
\end{gathered}
$$

sendo $S_{1}=\int_{R-\Delta}^{R+\Delta} d \rho\left(\frac{1}{2}\left(\frac{d \tilde{\varphi}}{d \rho}\right)^{2}+U\right)$.

Podemos obter $\mathrm{R}$ minimizando a ação

$$
\frac{d S_{e}}{d R}=-2 \pi^{2} R^{3} \epsilon+6 \pi^{2} R^{2} S_{1}=0,
$$




$$
R=3 S_{1} / \epsilon
$$

Portanto,

$$
S_{1}=\frac{1}{3} \epsilon R
$$

Vemos que $\mathrm{R} \rightarrow \infty$ quando $\epsilon \rightarrow 0$. Por isso, na nossa aproximação, desprezamos o termo de atrito quando $\epsilon$ é muito pequeno, pois $R$ fica muito grande e o termo de atrito tem $R$ no denominador.

Usando a expressão de $S_{1}$, a ação (149) pode ser escrita como

$$
S_{e}=\frac{27}{2 \epsilon^{3}} \pi^{2} S_{1}^{4}
$$

Em nossa aproximação podemos escrever a equação de movimento do campo escalar desprezando o termo de atrito,

$$
\ddot{\varphi}=V^{\prime}(\varphi),
$$

onde o ponto indica derivada com relação a $\rho$. Podemos multiplicar os dois lados por $\dot{\varphi}$,

$$
\ddot{\varphi} \dot{\varphi}=V^{\prime}(\varphi) \dot{\varphi} \text {. }
$$

Integrando os dois lados com relação a $\rho$ obtemos

$$
\int \ddot{\varphi} \dot{\varphi} d \rho=\frac{\dot{\varphi}^{2}}{2}+c t e=\int V^{\prime}(\varphi) \dot{\varphi} d \rho=\int \frac{d V}{d \varphi} \frac{d \varphi}{d \rho} d \rho=V(\varphi)+c t e,
$$

ou seja,

$$
V=\frac{\dot{\varphi}^{2}}{2}+c t e
$$

Deste modo,

$$
\dot{\varphi}=\sqrt{2 V}+\text { cte. }
$$

Vemos que a constante equivale a uma "velocidade inicial", que no nosso caso é zero, portanto,

$$
\dot{\varphi}=\sqrt{2 V} .
$$


Considerando $\epsilon$ pequeno podemos aproximar esta equação por

$$
\dot{\varphi}=\sqrt{2 U}
$$

Podemos então substituir $\dot{\varphi}$ no termo cinético da expressão de $S_{1}$ de modo que

$$
S_{1}=\int d \rho\left(\frac{1}{2}\left\{\left(\frac{d \tilde{\varphi}}{d \rho}\right) \sqrt{2 U}\right\}+U\right)
$$

que pode ser escrito como

$$
S_{1}=\int_{\varphi_{-}}^{\varphi+} d \varphi \frac{1}{2} \sqrt{2 U}+\int U d \rho .
$$

Por outro lado, $U(\varphi)$ pode ser escrito na forma

$$
U=\sqrt{U} \frac{1}{\sqrt{2}} \frac{d \varphi}{d \rho} .
$$

Integrando os dois lados desta equação com relação a $\rho$, obtemos

$$
\int U d \rho=\int \sqrt{U} \frac{1}{\sqrt{2}} d \varphi
$$

Substituindo a expressão acima no segundo termo da expressão de $S_{1}$ obtemos

$$
S_{1}=\sqrt{2} \int_{\varphi_{-}}^{\varphi_{+}} d \varphi \sqrt{U}
$$

Substituindo o nosso potencial supersimétrico de Wess-Zumino $U=\mid 2 m \varphi-$ $\left.3 \lambda \varphi^{2}\right|^{2}$

$$
\begin{gathered}
S_{1}=\sqrt{2} \int_{0}^{\frac{2 m}{3 \lambda}} d \varphi\left\{2 m \varphi-3 \lambda \varphi^{2}\right\} \\
S_{1}=\sqrt{2}\left\{4 m^{3} / 27 \lambda^{2}\right\} .
\end{gathered}
$$

Agora, podemos estabelecer uma condição mais precisa para que o atrito seja desprezível [53],

$$
\frac{w R}{3} \gg 1
$$


que pode ser escrita em função de $S_{1}$ como

$$
\frac{w S_{1}}{\epsilon} \gg 1
$$

onde $w=\left\{U^{\prime \prime}\left(\varphi_{+}\right)\right\}^{\frac{1}{2}}=\sqrt{8} m$.

Substituindo o valor que encontramos para $S_{1}$ obtemos a condição

$$
\sqrt{8} m\left\{4 m^{3} / 27 \lambda^{2} \epsilon\right\} \gg 1 \text {. }
$$

Usando os valores $\lambda=10^{-2}$ e $\epsilon=10^{-47} \mathrm{GeV}^{4}$, obtemos para a massa a condição

$$
m^{4} \gg \sim 10^{-51} \mathrm{GeV}^{4}
$$

ou seja,

$$
m \gg \sim 10^{-13} \mathrm{GeV} \text {. }
$$

Assim, para toda massa maior que um certo fator (de 1 a 9 ), vezes $10^{-13} \mathrm{GeV}$, a aproximação de parede fina pode ser considerada uma boa aproximação. Como só queremos estimar uma ordem de grandeza para o resultado não precisamos saber qual o fator exato, mas supomos que iremos só até este limite em que a aproximação é válida. Como vimos no início do capítulo, este valor de massa corresponde ao limite em que a altura da barreira do potencial fica da mesma ordem de grandeza do potencial no mínimo metaestável. Como apenas queremos estimar uma ordem de grandeza para o nosso resultado, esta aproximação será suficiente para nossos fins.

Como vimos, a ação Euclideana é dada por

$$
S_{e}=\frac{27}{2 \epsilon^{3}} \pi^{2} S_{1}^{4}
$$

Usando a expressão que obtivemos para $S_{1}$ obtemos

$$
S_{e}=\frac{m^{12}}{\epsilon^{3} 10^{2} \lambda^{8}}
$$

Usando-se $\epsilon=10^{-47} \mathrm{GeV}$,

$$
S_{e}=10^{140}\left(\frac{m^{12}}{\lambda^{8}}\right)
$$

Para $\lambda=10^{-2}$ obtemos 


$$
S_{e}=10^{156} m^{12}
$$

Portanto, a expressão da taxa de decaimento por unidade de volume fica com a forma

$$
\frac{\Gamma}{V}=\frac{\hbar\left(10^{156} m^{12}\right)^{2}}{(2 \pi \hbar)^{2}} \cdot e^{-10^{156} m^{12}} \cdot\left(\frac{\operatorname{det}^{\prime}\left(-\partial_{\mu} \partial_{\mu}+V^{\prime \prime}(\bar{\varphi}(\rho))\right.}{\operatorname{det}\left(-\partial_{\mu} \partial_{\mu}+V^{\prime \prime}(2 m / 3 \lambda)\right)}\right)^{-\frac{1}{2}} .
$$

O limite semiclássico é válido sempre que $|S| \gg \hbar$. Como estamos trabalhando nas unidades naturais em que $\hbar=1$, esta condição equivale a $|S| \gg 1$. Esta condição será válida em nosso caso sempre que

$$
10^{156} m^{12} \gg 1
$$

que equivale à condição para a massa

$$
m \gg \sim 10^{-13} \mathrm{GeV} \text {. }
$$

Isto corresponde à mesma ordem de grandeza em que nossa aproximação é válida. Isto ocorre pois no nosso caso $\epsilon$ é muito pequeno.

Até agora estivemos desprezando em nossos cálculos o efeito da gravitação, tanto que a mesma não foi considerada em nossa ação. É possível mostrar que em um certo domínio este efeito é de fato desprezível, e junto com ele também o efeito da expansão do universo. No entanto como está demonstrado no Apêndice $\mathrm{C}$, nos casos em que a massa da partícula é bem maior que $10^{-2,8} \mathrm{GeV}$ o efeito gravitacional passa a não ser mais desprezível. Nos casos em que a massa da partícula é da ordem de $10^{-2,8} \mathrm{GeV}$, recuperamos nosso resultado original a menos de um fator de 10. Para qualquer massa $m \gg 10^{-2,8} \mathrm{GeV}$, (para $\lambda=10^{-2}$ ), incluindo a gravitação, obtemos formalmente para a taxa de decaimento a expressão

$$
\frac{\Gamma}{V}=\frac{\hbar\left(10^{122}\right)^{2}}{(2 \pi \hbar)^{2}} \cdot\left(\frac{\operatorname{det}^{\prime}\left(-\partial_{\mu} \partial_{\mu}+V^{\prime \prime}(\varphi(\rho))\right.}{\operatorname{det}\left(-\partial_{\mu} \partial_{\mu}+V^{\prime \prime}(2 m / 3 \lambda)\right)}\right)^{-\frac{1}{2}} \cdot e^{-10^{122}} .
$$

(Ver o cálculo no Apêndice C).

Como o termo exponencial da taxa de decaimento domina sempre que estivermos dentro da validade do limite semiclássico, o termo pré-exponencial alterará o resultado de forma insignificante na ordem de grandeza em que 
estamos trabalhando, portanto uma simples estimativa da sua ordem de grandeza é suficiente. Sabemos que a dimensão do termo pré-exponencial é $m^{4}$ e seu valor é determinado pelos parâmetros da teoria que têm dimensão de massa, que são $\varphi(2 m / 3 \lambda), \sqrt{V^{\prime \prime}(2 m / 3 \lambda)} \mathrm{e} R^{-1}$. Portanto [59]

$$
\frac{\hbar S\left(\varphi_{0}\right)^{2}}{(2 \pi \hbar)^{2}} \cdot\left(\frac{\operatorname{det}^{\prime}\left(-\partial_{\mu} \partial_{\mu}+V^{\prime \prime}\left(\varphi_{0}(\rho)\right)\right.}{\operatorname{det}\left(-\partial_{\mu} \partial_{\mu}+V^{\prime \prime}(2 m / 3 \lambda)\right)}\right)^{-\frac{1}{2}}=O\left\{R^{-4}, \varphi^{4}, V^{\prime \prime 2}(2 m / 3 \lambda)\right\}
$$

Para massas na faixa de $10^{-2,8} \mathrm{GeV}$ a $1000 \mathrm{GeV}$ por exemplo, que inclui desde a massa a partir da qual a gravidade se torna importante até a massa de um possível parseiro supersimétrico do neutralino, o logaritmo de todas estas grandezas $\left(R^{-4}, \varphi^{4}, V^{\prime \prime 2}(2 m / 3 \lambda)\right)$ têm magnitude na ordem de $10^{1}$ a $10^{2}$ $\mathrm{GeV}$.

A expressão da taxa de decaimento que obtivemos corresponde ao número de partículas que decaem por unidade de tempo e volume. Invertendo esta expressão obtemos o tempo (vezes o volume) do decaimento de uma partícula. Se calcularmos a raiz quarta desta expressão encontramos então o tempo de decaimento da partícula, dado que em todo o cálculo consideramos uma simetria $\mathrm{O}(4)$ das coordenadas de espaço-tempo.

Calculando o logaritmo do tempo de decaimento para as ordens de grandeza de massa citadas acima obtemos

$$
4 \times \ln (\text { tempo de decaimento })\left(\mathrm{GeV}^{-4}\right)=10^{122} \pm 10^{2} \approx 10^{122}
$$

Exponenciando os dois lados da equação e calculando a raiz quarta da expressão, obtemos o tempo de decaimento

$$
t=e^{\frac{10^{122}}{4}} G e V^{-1} .
$$

Convertendo de $\mathrm{GeV}$ para segundo $\left(1 \mathrm{GeV}^{-1}=10^{-25}\right.$ segundos $)$, obtemos

$$
t=10^{-25} e^{\frac{10^{122}}{4}} \text { segundos, }
$$

que é muito maior que a idade do universo $=10^{17}$ segundos. Ou seja, se houver decaimento será para massas bem menores que $10^{-2,8} \mathrm{GeV}$.

Para massas pequenas o suficiente, no limite em que a gravidade não é importante (massas menores que $10^{-2,8} \mathrm{GeV}$ ), porém maiores que um certo fator vezes $10^{-13} \mathrm{GeV}$, vimos que a taxa de decaimento é dada pela expressão 


$$
\frac{\Gamma}{V}=\frac{\hbar\left(10^{156} m^{12}\right)^{2}}{(2 \pi \hbar)^{2}} \cdot e^{-10^{156} m^{12}} \cdot\left(\frac{\operatorname{det}^{\prime}\left(-\partial_{\mu} \partial_{\mu}+V^{\prime \prime}\left(\varphi_{0}(\rho)\right)\right.}{\operatorname{det}\left(-\partial_{\mu} \partial_{\mu}+V^{\prime \prime}(2 m / 3 \lambda)\right)}\right)^{-\frac{1}{2}} .
$$

Dentro da validade do limite semiclássico, o termo exponencial será sempre dominante e portanto o termo pré-exponencial causará uma alteração menor do que a de um fator de 2 multiplicando o expoente do termo dominante. Dentro da ordem de grandeza que obtivemos este fator de 2 não causará nenhuma diferença significante quando for multiplicado por $10^{156}$. Portanto poderíamos desprezar a contribuição do determinante se não fosse pelo fato de que ele é quem fornece a unidade dimensional na nossa expressão. Como estamos trabalhando com unidades de massa de $\mathrm{GeV}$ vamos apenas considerá-lo então como $1 \mathrm{GeV}^{4}$, o que simplificará os cálculos, e como vimos, não alterará o resultado.

Assim ficamos com a seguinte expressão para a taxa de decaimento para a faixa de massas citada acima,

$$
\frac{\Gamma}{V}=e^{-10^{156} m^{12}} G e V^{4}
$$

A partir desta expressão obtemos o seguinte tempo de decaimento,

$$
\text { tempo de decaimento }\left(G e V^{-1}\right)=\left\{\exp \left(10^{156} m^{12}\right)\right\}^{\frac{1}{4}} G e V^{-1} \text {. }
$$

Convertendo de GeV para segundo, obtemos que o tempo de decaimento é dado por

$$
\text { tempo de decaimento }(s)=10^{-25}\left\{\exp \left(10^{156} m^{12}\right)\right\}^{\frac{1}{4}} s \text {. }
$$

Podemos igualar este tempo de decaimento à idade do universo, que é igual à $10^{17}$ segundos,

$$
10^{-25}\left\{\exp \left(10^{156} \mathrm{~m}^{12}\right)\right\}^{\frac{1}{4}} s=10^{17} s .
$$

Rearranjando os termos e calculando o logaritmo dos dois lados da equação,

$$
\frac{10^{156} m^{12}}{4}=96,7 \approx 10^{2}
$$

Podemos ver que a massa de uma partícula de energia escura que poderia decair no tempo da idade do universo seria aproximadamente 


$$
m \sim 10^{-13} \mathrm{GeV} .
$$

Como havíamos calculado no início do capítulo, para este valor de massa a altura da barreira de potencial possui mesma ordem de grandeza do potencial no mínimo metaestável, ou seja, $10^{-47} \mathrm{GeV}^{4}$.

Podemos agora estimar quais termos de quebra de supersimetria poderiam gerar a densidade de energia da constante cosmológica no falso vácuo. Como $\varphi \sim \frac{2 m}{3 \lambda} \sim \frac{m}{\lambda}$ neste ponto, podemos ver que termos tais como

$$
Q(\varphi)=m^{2} \varphi^{2}, \lambda m \varphi^{3}, \lambda^{2} \varphi^{4},
$$

quebram a supersimetria e geram a densidade de energia que queremos no vácuo metaestável. 


\section{8 - Conclusão}

Foi calculado o decaimento de uma partícula de energia escura com massa da ordem de $10^{-13} \mathrm{GeV}$, descrita por um potencial $V(\varphi)=\mid 2 m \varphi-$ $\left.3 \lambda \varphi^{2}\right|^{2}+Q(\varphi)$, do mínimo metaestável para o mínimo estável de seu potencial. Vimos que o tempo deste processo é compatível com o tempo de vida do universo, e portanto um modelo de quintessência como este pode fornecer um mecanismo, a partir de teoria de campos, para explicar o decaimento da energia escura em matéria escura, aliviando certos problemas do modelo padrão da cosmologia. 


\section{Apêndice A: A Equação de Movimento de $\phi$}

A ação do campo escalar correspondente à quintessência é dada por

$$
S=\int \sqrt{-g}\left(-\frac{1}{2} \partial_{\mu} \phi \partial^{\mu} \phi-V(\phi)\right)
$$

Podemos obter a equação de movimento para este campo usando o princípio de mínima ação, ou seja impondo $\delta S=0$. Para isto, vamos calcular a variação da ação,

$$
\delta S=\int d^{4} x \delta(\sqrt{-g} L)
$$

Como $S=S\left(\phi, \partial_{\mu} \phi\right)$ devemos variar a ação da forma

$$
\delta(\sqrt{-g} L)=\frac{\partial(\sqrt{-g} L)}{\partial \phi} \delta \phi+\frac{\partial(\sqrt{-g} L)}{\partial \partial_{\mu} \phi} \delta \partial_{\mu} \phi .
$$

Sabendo que

$$
\delta \partial_{\mu} \phi=\partial_{\mu} \delta \phi
$$

obtemos

$$
\delta(\sqrt{-g} L)=\frac{\partial(\sqrt{-g} L)}{\partial \phi} \delta \phi+\partial_{\mu}\left(\frac{\partial(\sqrt{-g} L)}{\partial \partial_{\mu} \phi} \delta \phi\right)-\partial_{\mu}\left(\frac{\partial(\sqrt{-g} L)}{\partial \partial_{\mu} \phi}\right) \delta \phi .
$$

Podemos eliminar o termo de superfície. Fazendo isto, inserimos o resultado na ação e igualamos a zero, obtendo assim

$$
\delta S=\int d^{4} x\left[\frac{\partial(\sqrt{-g} L)}{\partial \phi}-\frac{\partial_{\mu}(\partial(\sqrt{-g} L))}{\partial \partial_{\mu} \phi}\right] \delta \phi=0,
$$

portanto

$$
\begin{aligned}
& {\left[\frac{\partial(\sqrt{-g} L)}{\partial \phi}-\frac{\partial_{\mu}(\partial(\sqrt{-g} L))}{\partial \partial_{\mu} \phi}\right]=0,} \\
& {\left[\sqrt{-g} \frac{\partial(L)}{\partial \phi}-\partial_{\mu}\left(\sqrt{-g} \frac{\partial L}{\partial \partial_{\mu} \phi}\right)\right]=0,}
\end{aligned}
$$




$$
\sqrt{-g} \frac{\partial V}{\partial \phi}-\partial_{\mu} \sqrt{-g}\left(\partial^{\mu} \phi\right)-\sqrt{-g} \partial_{\mu} \partial^{\mu} \phi=0
$$

Como $\sqrt{-g}=a^{3}(t)$ obtemos,

$$
a^{3} \frac{\partial V}{\partial \phi}+3 a^{2} \dot{a} \dot{\phi}+a^{3} \ddot{\phi}=0
$$

Dividindo tudo por $a^{3}$ e substituindo $H=\frac{\dot{a}}{a}$, obtemos finalmente a equação de movimento do campo $\phi$,

$$
\frac{\partial V}{\partial \phi}+3 H \dot{\phi}+\ddot{\phi}=0
$$




\section{Apêndice B- Equação de Movimento de $\phi$ no Es- paço Euclideano}

A ação do campo $\phi$ é dada por

$$
S_{E}(\phi(x))=\int d t d^{3} x\left(\frac{1}{2} \partial_{\mu} \phi(x) \partial_{\mu} \phi(x)+V(\phi(x))\right) .
$$

Para encontrar a equação de movimento variamos a ação com relação a $\phi$ e igualamos a zero de acordo com o princípio da mínima ação,

$$
\frac{\delta S e(\phi(x))}{\delta \phi}=\left(-\partial_{\mu} \partial_{\mu} \phi(x)+V^{\prime}(\phi)\right)=0
$$

onde $\mu$ representa as 3 coordenadas espaciais e a coordenada temporal,

$$
-\partial_{\mu} \partial_{\mu} \phi=-\partial_{x} \partial_{x} \phi-\partial_{y} \partial_{y} \phi-\partial_{z} \partial_{z} \phi-\partial_{\tau} \partial_{\tau} \phi
$$

Devido à simetria euclideana do problema podemos assumir um campo da forma $\phi(\vec{x}, \tau) \rightarrow \phi\left(\left(|\vec{x}|^{2}+\tau^{2}\right)^{\frac{1}{2}}\right)=\phi(\rho)$. Neste caso,

$$
\begin{gathered}
-\partial_{x} \partial_{x} \phi=-\partial_{x}\left(\frac{\partial \phi}{\partial \rho} \frac{\partial \rho}{\partial x}\right)=-\partial_{x}\left(\frac{\partial \phi}{\partial \rho} \frac{x}{\rho}\right) \\
=\frac{x^{2}}{\rho^{2}} \frac{\partial^{2} \phi}{\partial \rho^{2}}+\frac{\partial \phi}{\partial \rho} \frac{1}{\rho}-\frac{\partial \phi}{\partial \rho} \frac{x^{2}}{\rho^{3}}
\end{gathered}
$$

Para a coordenada temporal os cálculos são análogos. Assim obtemos

$$
-\partial_{\tau} \partial_{\tau}=\frac{\tau^{2}}{\rho^{2}} \frac{\partial^{2} \phi}{\partial \rho^{2}}+\frac{\partial \phi}{\partial \rho} \frac{1}{\rho}-\frac{\partial \phi}{\partial \rho} \frac{\tau^{2}}{\rho^{3}} .
$$

Somando a contribuição das três coordenadas espaciais mais a temporal obtemos

$$
\frac{\partial^{2} \phi}{\partial \rho^{2}}+4 \frac{\partial \phi}{\partial \rho} \frac{1}{\rho}-\frac{\partial \phi}{\partial \rho} \frac{1}{\rho}
$$

que nos fornece

$$
\frac{\partial^{2} \phi}{\partial \rho^{2}}+\frac{\partial \phi}{\partial \rho} \frac{3}{\rho}
$$


Portanto a equação de movimento do campo escalar é

$$
\frac{\partial^{2} \phi}{\partial \rho^{2}}+3 \frac{\partial \phi}{\partial \rho} \frac{1}{\rho}-V^{\prime}(\phi)=0 .
$$




\section{Apêndice C: A Inclusão da Gravidade}

Incluindo a gravitação, a ação do campo escalar é dada por

$$
\bar{S}=\int d^{4} x \sqrt{-g}\left(\frac{1}{2} g^{\mu \nu} \partial_{\mu} \varphi \partial_{\nu} \varphi-V(\varphi)-\frac{R}{16 \pi G}\right),
$$

onde R é o escalar de curvatura. Chamaremos as grandezas calculadas para o caso sem gravitação de $R_{0}, S_{0}$, etc, enquanto que as grandezas calculadas incluindo-se o efeito gravitacional serão denotadas por $\bar{R}, \bar{S}$, etc.

No nosso problema podemos considerar a simetria esférica, o que simplificará bastante os cálculos. Podemos definir coordenadas angulares em cada 3-esfera que comporá a nossa variedade. Definimos a coordenada radial $\xi$ para medir distâncias ao longo das curvas radiais normais a cada 3-esfera. Assim, podemos escrever a métrica [58]

$$
d s^{2}=(d \xi)^{2}+\rho(\xi)^{2}(d \Omega)^{2}
$$

onde $\xi$ é a coordenada radial, $(d \Omega)^{2}$ é o elemento de distância em uma 3esfera unitária e $\rho$ é o raio de curvatura da 3-esfera. É possível notar que podemos redefinir $\xi$ adicionando-se uma constante sem alterar a forma da métrica, ou seja, podemos escolher o início da medida de $\xi$ arbitrariamente.

Com a métrica acima podemos calcular os símbolos de Christoffel e obter a equação de movimento do campo escalar $\varphi$ no espaço euclideano [58]

$$
\varphi^{\prime \prime}+\frac{3}{\rho} \cdot \rho^{\prime} \varphi^{\prime}=\frac{d U}{d \varphi}
$$

onde ${ }^{\prime}=\frac{\partial}{\partial \xi}$.

Com a mesma métrica obtemos que a equação de Einstein

$$
G_{\xi \xi}=-8 \pi G T_{\xi \xi}
$$

se torna [58]

$$
\rho^{\prime 2}=1+\frac{1}{3} k \rho^{2}\left(\frac{1}{2} \varphi^{\prime 2}-V\right),
$$

onde $k=8 \pi G$. As outras equações de Einstein são identidades ou são consequências triviais dessa equação. 
Com a métrica podemos calcular também o escalar de curvatura. Substituindo o resultado na ação, obtemos em ordem zero em $\epsilon$ [58]

$$
\bar{S}=2 \pi^{2} \int d \xi\left(\rho^{3}\left(\frac{1}{2} \varphi^{2}+V\right)+\frac{3}{k}\left(\rho^{2} \rho^{\prime \prime}+\rho \rho^{\prime 2}-\rho\right)\right) .
$$

Voltando à equação do campo escalar podemos ver que a mesma difere do caso sem gravitação em dois aspectos. Aqui a variável independente é $\xi$ ao invés de $\rho$, mas esta é uma alteração trivial. Além disso o coeficiente do termo $\varphi^{\prime}$ é $\frac{\rho^{\prime}}{\rho}$, enquanto no caso sem gravitação ele é $\frac{1}{\rho}$. No entanto, no limite da aproximação de pequenas diferenças de energia, este termo é desprezado tanto no caso com gravitação como no caso sem ela. Mas como este termo agora é diferente então o limite em que podemos usar essa aproximação é também diferente. Verificaremos este limite posteriormente. Por enquanto, assumiremos sua validade. Usando a aproximação a equação do campo escalar em ordem zero em $\epsilon$ fica

$$
\varphi^{\prime \prime}=\frac{d U}{d \varphi}
$$

Como no caso sem gravitação, porém, agora a derivada segunda de $\varphi$ é com relação a $\xi$ ao invés de $\rho$.

Podemos integrar esta equação e obter

$$
\left(\frac{1}{2} \varphi^{\prime 2}-U\right)=c t e .
$$

No nosso caso a constante corresponde a $U\left(\varphi_{+}\right)$. Como $\varphi(\infty)=\varphi_{+}$, que é o ponto de mínimo onde $U=0$,

$$
\left(\frac{1}{2} \varphi^{\prime 2}-U\right)=U\left(\varphi_{+}\right)=0
$$

Portanto,

$$
\frac{1}{2}\left(\frac{d \varphi}{d \xi}\right)^{2}=U
$$

Calculando a raiz quadrada,

$$
\frac{d \varphi}{d \xi}=\sqrt{2 U}
$$

Separando as variáveis e integrando obtemos que 


$$
\int_{\phi_{0}}^{\phi} d \varphi(2 U)^{-\frac{1}{2}}=\xi-\bar{\xi} .
$$

Como no caso sem gravitação porém substitui-se $\rho$ por $\xi . \bar{\xi}$ é uma constante de integração escolhida convencionalmente.

Tendo $\varphi$ podemos encontrar $\rho$ a partir da equação de Einstein. Para especificar a solução precisamos de uma constante de integração que será

$$
\bar{\rho}=\rho(\bar{\xi}) .
$$

$\bar{\rho}$ por sua vez possui um significado físico, correspondendo ao raio de curvatura da parede da bolha que separa o falso vácuo do verdadeiro, portanto o chamaremos aqui de $\bar{R}$.

Para encontrarmos $\bar{R}$, primeiro calculamos $\bar{S}$, a diferença entre a ação da solução oscilante e a ação do falso vácuo, depois encontramos $\bar{R}$ fazendo com que $\bar{S}$ seja estacionário sob variações de $\rho$, da mesma forma que havíamos feito pra $R$.

Na ação,

$$
\bar{S}=2 \pi^{2} \int d \xi\left(\rho^{3}\left(\frac{1}{2} \varphi^{2}+V\right)+\frac{3}{k}\left(\rho^{2} \rho^{\prime \prime}+\rho \rho^{\prime 2}-\rho\right)\right) .
$$

Podemos eliminar o termo de derivada segunda integrando por partes,

$$
\int d \xi\left(\rho^{2} \rho^{\prime \prime}\right)=\rho^{2} \rho^{\prime}-2 \int d \xi\left(\rho \rho^{2}\right) .
$$

O termo de superfície não importa pois estamos interessados na diferença entre a ação de duas soluções que se igualam no infinito.

Substituindo na ação, obtemos

$$
\bar{S}=2 \pi^{2} \int d \xi\left(\rho^{3}\left(\frac{1}{2} \varphi^{\prime 2}+V\right)-\frac{3}{k}\left(\rho \rho^{\prime 2}+\rho\right)\right) .
$$

Podemos usar a equação de Einstein,

$$
\rho^{\prime 2}=1+\frac{1}{3} k \rho^{2}\left(\frac{1}{2} \varphi^{\prime 2}-V\right)
$$

que pode ser escrita como 


$$
\frac{3}{k} \rho \rho^{\prime 2}=\frac{3 \rho}{k}+\rho^{3}\left(\frac{1}{2} \varphi^{\prime 2}-V\right),
$$

para eliminarmos o termo em $\rho^{\prime}$ na ação. Assim, obtemos a ação [58]

$$
\bar{S}=4 \pi^{2} \int d \xi\left(\rho^{3} V-\frac{3 \rho}{k}\right) .
$$

Podemos agora calcular $\bar{S}$ considerando a aproximação de parede fina, cuja validade será demonstrada posteriormente. Como antes, vamos dividir a ação em 3 partes: dentro da bolha, fora, e na parede. Fora da bolha a ação da solução oscilatória é igual ao potencial do falso vácuo. Assim como antes,

$$
\bar{S}_{\text {fora }}=0 .
$$

Na parede, onde $\rho=\bar{R}$, a diferença entre as duas ações é aproximadamente

$$
\bar{S}_{\text {parede }}=4 \pi^{2} \bar{R}^{3} \int d \xi U(\varphi)=2 \pi^{2} \bar{R}^{3} S_{1}
$$

onde $S_{1}$ é obtido analogamente ao caso sem gravitação substituindo-se porém $\rho$ por $\xi$.

Dentro da bolha, $\varphi$ é constante, e a equação de Einstein fica

$$
\left(\frac{d \rho}{d \xi}\right)^{2}=1-\frac{1}{3} k \rho^{2} V .
$$

Separando as variáveis e extraindo a raiz,

$$
d \xi=d \rho\left(1-\frac{1}{3} k \rho^{2} V\right)^{-\frac{1}{2}} .
$$

O integrando da nossa expressão da ação pode ser escrito como

$$
\left(\rho^{3} V-\frac{3 \rho}{k}\right)=-\frac{3 \rho}{k}\left(1-\frac{1}{3} k \rho^{2} V\right) .
$$

Substituindo as duas equações acima na expressão da ação, obtemos

$$
\bar{S}=4 \pi^{2} \int d \rho\left(1-\frac{1}{3} k \rho^{2} V\right)^{-\frac{1}{2}} \cdot \frac{-3 \rho}{k}\left(1-\frac{1}{3} k \rho^{2} V\right),
$$

e a expressão da ação da solução oscilatória menos a ação da solução estacionária na região interna da bolha é igual a 
$\bar{S}=\left\{-\frac{12 \pi^{2}}{k} \int_{0}^{\bar{\rho}} d \rho . \rho\left(1-\frac{1}{3} k \rho^{2} V\left(\varphi_{-}\right)\right)^{\frac{1}{2}}\right\}-\left\{-\frac{12 \pi^{2}}{k} \int_{0}^{\bar{\rho}} d \rho . \rho\left(1-\frac{1}{3} k \rho^{2} V\left(\varphi_{+}\right)\right)^{\frac{1}{2}}\right\}$

Após integrar, obtemos

$$
\bar{S}_{\text {dentro }}=\frac{12 \pi^{2}}{k^{2}} \frac{\left\{\left(1-\frac{1}{3} k \bar{\rho}^{2} V\left(\varphi_{-}\right)\right)^{\frac{3}{2}}-1\right\}}{V\left(\varphi_{-}\right)}-\frac{12 \pi^{2}}{k^{2}} \frac{\left\{\left(1-\frac{1}{3} k \bar{\rho}^{2} V\left(\varphi_{+}\right)\right)^{\frac{3}{2}}-1\right\}}{V\left(\varphi_{+}\right)} .
$$

Podemos calcular o raio $\bar{R}$ forçando $\bar{S}$ a ser estacionário,

$$
\frac{d \bar{S}(\rho)}{d \rho}=6 \pi^{2} \bar{R}^{2} S_{1}-\frac{12 \pi^{2}}{k^{2}}\left\{\left(1-\frac{1}{3} k \bar{R}^{2} V\left(\varphi_{-}\right)\right)^{\frac{1}{2}}\right\} k \bar{R}+\frac{12 \pi^{2}}{k^{2}}\left\{\left(1-\frac{1}{3} k \bar{R}^{2} V\left(\varphi_{+}\right)\right)^{\frac{1}{2}} k \bar{R}\right\}=0
$$

Substituindo-se $V\left(\varphi_{+}\right)=\epsilon, V\left(\varphi_{-}\right)=0$ e dividindo tudo por $\bar{R}$,

$$
6 \pi^{2} \bar{R} S_{1}-\frac{12 \pi^{2}}{k}+\frac{12 \pi^{2}}{k}\left\{\left(1-\frac{1}{3} k \bar{R}^{2} \epsilon\right)\right)^{\frac{1}{2}}=0 .
$$

Passando para o lado direito o terceiro termo e elevando os dois lados da equação ao quadrado, obtemos

$$
36 \pi^{4} \bar{R}^{2} S_{1}^{2}+\frac{144 \pi^{4}}{k^{2}}-\frac{144 \pi^{4} \bar{R} S_{1}}{k}=\frac{144 \pi^{4}}{k^{2}}\left(1-\frac{k \bar{R}^{2} \epsilon}{3}\right) .
$$

Cancelando o segundo termo do lado esquerdo com o primeiro do lado direito ficamos com

$$
36 \pi^{4} \bar{R}^{2} S_{1}^{2}+-\frac{144 \pi^{4} \bar{R} S_{1}}{k}=\frac{-48 \pi^{4}}{k} \frac{\bar{R}^{2} \epsilon}{} .
$$

Dividindo tudo por $\bar{R}$ e isolando $\bar{R}$ obtemos

$$
\bar{R}=\frac{144 S_{1}}{48 \epsilon+36 S_{1}^{2} k}=\frac{12 S_{1}}{4 \epsilon+3 S_{1}^{2} k} .
$$

$3 S_{1} / \epsilon=R_{0}$ é o raio da bolha na ausência de gravidade. O valor $\sqrt{\frac{3}{k \epsilon}}$ será denotado por $\Delta$. Substituindo estas expressões obtemos

$$
\bar{R}=\frac{12 S_{1}}{4 \epsilon+3 S_{1}^{2} k}=\frac{R_{0}}{\left(1+\left(\frac{R_{0}}{2 \Delta}\right)^{2}\right)} .
$$


Havíamos obtido que no caso sem gravitação a ação é dada por

$$
S_{0}=\frac{1}{6} \epsilon \pi^{2} R_{0}^{4}
$$

Comparando a equação antiga de $S_{0}$ do caso sem gravitação com a nova ação $\bar{S}$, e usando a relação entre $\bar{R}$ e $R_{0}$, podemos obter a relação entre a ação com gravitação e sem gravitação [58],

$$
\bar{S}=\frac{S_{0}}{\left(1+\left(\frac{R_{0}}{2 \Delta}\right)^{2}\right)^{2}} .
$$

Podemos agora conferir o limite de validade da nossa aproximação de parede fina. No caso sem gravitação o termo de atrito era $\frac{\varphi^{\prime}}{\rho}$ e o desprezávamos pois na parede da bolha esse termo é igual a $\frac{\varphi^{\prime}}{R}$ e $R_{0} \gg \varphi^{\prime}$. Vimos que podíamos usar a aproximação para uma massa da partícula de energia escura maior que $10^{-13} \mathrm{GeV}$. Agora no caso com gravitação $\frac{\varphi^{\prime}}{\rho}$ é substituído por $\frac{\rho^{\prime} \varphi^{\prime}}{\rho}$, então é esta quantidade que deve ser muito pequena na parede.

Dividindo a expressão (229) vinda da equação de Einstein por $\rho^{2}$ obtemos

$$
\frac{\rho^{2}}{\rho^{2}}=\frac{1}{\rho^{2}}+\frac{1}{3} k\left(\frac{1}{2} \varphi^{\prime 2}-V\right) .
$$

O lado esquerdo dessa equação será pequeno se os dois termos do lado direito forem pequenos. O primeiro termo é o mesmo que o do caso sem gravitação. $\mathrm{O}$ termo entre parênteses no segundo termo tem magnitude 0 dentro da bolha, e tem magnitude $\epsilon=10^{-47} \mathrm{GeV}^{4}$ fora da bolha. Então na parede ele é certamente menor que $\epsilon$. Podemos então superestimá-lo substituindo-o por $\epsilon$. Assim o segundo termo fica igual a $\frac{k \epsilon}{3}=\frac{1}{\Delta^{2}}$. Podemos então usar a aproximação de pequenas energias se tanto $\bar{R}$ quanto $\Delta$ forem grandes comparados à variação de $\varphi$. No caso sem gravitação, como vimos, nossa condição para $R_{0}$ correspondia a

$$
\frac{w R_{0}}{3} \gg 1
$$

No caso com gravitação $R_{0}$ é substituído por $\bar{R}$ de acordo com a equação (245). Vamos encontrar então o valor da expressão (245) começando pelo denominador. Como $R_{0} \sim \frac{m^{3} 10^{47}}{\lambda^{2}}$, e sabendo que $\Delta=\sqrt{\frac{3}{k \epsilon}}=10^{42,5} \mathrm{GeV}^{-1}$,

$$
\frac{R_{0}^{2}}{\Delta^{2}}=\frac{m^{6} 10^{9}}{\lambda^{4}}
$$


Para verificar a validade da nossa aproximação consideraremos o caso em que $\frac{R_{0}^{2}}{\Delta^{2}} \gg 1$, pois caso contrário obtemos exatamente os resultados originais sem gravitação. Neste caso obtemos para $\bar{R}$

$$
\bar{R}=\frac{R_{0}}{\left(\frac{m^{6} 10^{9}}{\lambda^{4}}\right)} .
$$

Substituindo o $R_{0}$ que havíamos obtido no caso sem gravitação,

$$
\bar{R} \sim \frac{m^{3}}{\lambda^{2} \epsilon} \cdot \frac{\lambda^{4}}{m^{6} 10^{9}} \sim \frac{10^{34}}{m^{3}} .
$$

Portanto a condição de validade da nossa aproximação sobre $\bar{R}$ agora é

$$
\frac{w}{3} \frac{10^{34}}{m^{3}} \gg 1
$$

Como vimos anteriormente $w=\sqrt{8} m$, então,

$$
\begin{aligned}
& \frac{\sqrt{8} m}{3} \frac{10^{34}}{m^{3}} \gg 1, \\
& \sim \frac{10^{34}}{m^{2}} \gg 1 .
\end{aligned}
$$

Portanto obtemos a seguinte condição para a massa,

$$
m \ll 10^{17},
$$

o que contempla todos os casos de interesse. Além disso temos agora também a mesma condição para $\Delta$,

$$
\frac{w \Delta}{3} \gg 1
$$

que corresponde a

$$
\sim m 10^{42,5} \gg 1
$$

ou seja,

$$
m \gg 10^{-42,5},
$$

o que mostra que a aproximação de parede fina que usamos aqui é uma boa aproximação para todos os casos de interesse. 
Analisando a expressão da nova ação podemos observar que para valores da razão $\frac{R_{0}}{\Delta}$ muito menores que 1 , recuperamos nosso resultado original (sem gravitação), e para valores da ordem de 1 temos o resultado original a menos de um fator de 10 .

Quando ocorre um decaimento de um vácuo metaestável que tem uma densidade de energia $\epsilon$ para um vácuo com densidade de energia zero, há uma liberação de energia proporcional à densidade de energia do vácuo metaestável e ao volume da bolha de novo vácuo formada. Há, portanto, um raio de Schwarzschild associado a esta energia.

Uma esfera de raio $\mathrm{R}$ e densidade de energia $\epsilon$ tem um raio de Schwarzschild dado por

$$
r_{s}=2 G \epsilon\left(\frac{4 \pi R^{3}}{3}\right),
$$

onde $G$ é a constante gravitacional, igual a $\frac{1}{m_{p}^{2}}=10^{-38} \mathrm{GeV}^{-2}$.

Para acharmos o raio $R$ da esfera no qual este raio de Schwarzschild fica da ordem do próprio raio $R$, igualamos $r_{s}$ ao raio da esfera:

$$
R=2 G \epsilon\left(\frac{4 \pi R^{3}}{3}\right)
$$

Isolando $R$,

$$
\begin{aligned}
& R^{2}=\frac{3}{8 \pi G \epsilon}, \\
& R=\sqrt{\frac{3}{8 \pi G \epsilon}} .
\end{aligned}
$$

Vemos que este valor corresponde justamente o valor $\triangle$. Esferas com raio maior que este valor, terão o seu raio de Schwarzschild maior que o raio da esfera, pois o mesmo aumenta com $R^{3}$. Como havíamos visto, no limite em que a razão $\frac{R_{0}}{\Delta}$ é muito maior que 1 é importante considerar nos cálculos o efeito da gravitação. Isto significa que o efeito gravitacional se torna importante a partir do momento em que o raio da bolha de novo vácuo fica da ordem do raio de Shwarzschild associado à energia liberada na transição.

Foi calculado, no texto, que o raio da bolha de novo vácuo formada é dado por

$$
R_{0}=\frac{3 S_{1}}{\epsilon}
$$


No caso em que estamos trabalhando, $\epsilon$ é a constante cosmológica que é igual à $10^{-47} \mathrm{GeV}^{4}$ e $S_{1}$ é dado por

$$
S_{1}=\sqrt{2}\left\{4 m^{3} / 27 \lambda^{2}\right\} .
$$

Deste modo,

$$
R_{0} \sim \frac{12 m^{3}}{27 \lambda^{2} \epsilon} .
$$

O raio da bolha de novo vácuo será igual ao raio de Schwarzschild quando

$$
R_{0}=\sqrt{\frac{3}{8 \pi G \epsilon}}=\sqrt{\frac{3}{8 \pi 10^{-38} 10^{-47}}} \sim 10^{42,5} \mathrm{GeV}^{-1} .
$$

Para sabermos qual massa corresponde, no nosso caso, à formação de uma bolha com este raio, usamos a expressão de $R_{0}$,

$$
R_{0}=\frac{12 m^{3}}{27 \lambda^{2} \epsilon}=\frac{12 m^{3}}{27 . \lambda^{2} 10^{-47}}=10^{42,5} \mathrm{GeV}^{-1} .
$$

Para $\lambda=10^{-2}$ obtemos que a massa é

$$
m=10^{-2,8} \mathrm{GeV} .
$$

Portanto, para raios maiores que $10^{42,5} \mathrm{GeV}^{-1}$, o que corresponde a massas maiores que $10^{-2,8} \mathrm{GeV}$, o raio de Schwarzschild é maior que o raio da bolha. Neste caso nossa expressão da ação é consideravelmente alterada pela gravitação.

Se fizermos uma extensão do nosso resultado (247) para os casos em que $m>10^{-2,8} \mathrm{GeV}$, obtemos

$$
\bar{S}=\frac{S_{0}}{\frac{m^{12} 10^{18}}{\lambda^{8}}}=\frac{m^{12} 10^{140}}{\lambda^{8}} \cdot\left(\frac{\lambda^{8}}{m^{12} 10^{18}}\right)=10^{122},
$$

onde o fator 1 no denominador foi desprezado. Como vemos, a ação aqui independe do valor da massa e de $\lambda$, o que nos mostra uma perda de sensibilidade do resultado. Vemos que, de fato, o falso vácuo é estabilizado para valores grandes de massa (acima de $10^{-2,8} \mathrm{GeV}$ neste caso). O valor obtido de $\bar{S}$ nos leva a um tempo de decaimento da ordem de

$$
e^{\frac{10^{122}}{4}} \mathrm{GeV}^{-1}=e^{\frac{10^{122}}{4}} 10^{-25} \text { segundos, }
$$


que é muito maior que a idade do universo. Podemos ver que se houver uma partícula de energia escura que decaia durante o tempo da idade do universo segundo o nosso modelo, esta partícula terá massa muito menor que $10^{-2,8} \mathrm{GeV}$. 


\section{Referências Bibliográficas}

[1] L. D. Landau, "Teoria Clássica de Campos", Editorial Reverte, S. A. (1973)

[2] S. Weinberg, Rev. Mod. Phys. 61, 1-23 (1989).

[3] A. Friedmann, Z. Phys. 10, 377 (1922); ibid 21, 326 (1924).

[4] H. P. Robertson, Astrophys. J. 82, 284 (1935); ibid 83, 187- 257 (1936).

[5] A. G. Walker, Proc. Lond. Math. Soc. (2) 42, 90 (1936).

[6] S. M. Carroll, Living Rev. Relativity 4, 1 (2001).

[7] D. N. Spergel et. al., Astrophys. J. Suppl. 148, 175-194 (2003).

[8] J. Copeland, M. Sami, S. Tsujikawa, Int. Journal of Modern Physics D 15, 1753-1938 (2006).

[9] J. Frieman et al., Phys. Rev. Lett. 75, 2077 (1995)

[10] F. Lucchin, S. Matarrese, Phys. Rev. D 32, 1316 - 1322 (1985)

[11] P. Brax, J. Martin, Phys. Rev. D 61,103502 (2000)

[12] T. Barreiro, E. J. Copeland, N. J. Nunes, Phys. Rev. D 61, 127301 (2000)

[13] P. G. Bergmann, Int. J. Theor. Phys. 1, 2536 (1968).

[14] B. N. Breizman, V. T. Gurovich, V. P. Sokolov, Zh. Eksp. Teor. Fiz. 59, 288 (1970); Sov. Phys. JETP 32, 155 (1971).

[15] H. A. Buchdahl, Mon. Not. R. Astron. Soc. 150, 18 (1970). 
[16] T. V. Ruzmaikina, A. A. Ruzmaikin, Zh. Eksp. Teor. Fiz. 57, 680 (1969); Sov. Phys. JETP 30, 372 (1970).

[17] M. E. Peskin, D. V. Schroeder, "An Introduction to Quantum Field Theory", Addison-Wesley Publishing Company (1995).

[18] A. Friaca, J. S. Alcaniz, J. A. S. Lima, Mon. Not. Roy. Astron. Soc. 362, 1295 (2005).

[19] T. Clemson et. al., 1109.6234 [astro-ph], (2011).

[20] J. Valiviita, E. Majerotto, R. Maartens, JCAP 07, 020 (2008); J. H. He, B. Wang, E. Abdalla, Phys. Lett. B 671, 139 (2009).

[21] C. Feng, B. Wang, E. Abdalla, R. K. Su, Phys Lett B 665, 111 (2008).

[22] J. H. He, B. Wang, P. Zhang, Phys. Rev. D 80, 063530 .

[23] J. H. He, B. Wang, JCAP 06, 010 (2008).

[24] G. Caldera-Cabral, R. Maartens, B. Schaefer, JCAP 0907, 027 (2009).

[25] E. Abdalla, L. Raul W. Abramo, J. Souza, Phys. Rev. D 82, 023508 (2010).

[26] B. Wang, J.Zang, C. Y. Lin, E. Abdalla, S. Micheletti, Nucl. Phys. B 69, 778 (2007).

[27] E. Abdalla, R. Abramo, L. Sodre, B. Wang, Phys. Lett. B 673, 107-110 (2009).

[28] J. Lee, 1008.4620 [astro-ph], (2010) .

[29] M. Baldi, J. Lee, A. V. Maccio, Astroph. J. 732, 112 (2011).

[30] C. E. Pellicer et. al., 1102.5113v2 [astro-ph], (2011).

[31] Wang Bin, Gong Yun-Gui, Elcio Abdalla, Phys. Lett. B 624, 141-146 (2005); Wang Bin, Lin Chi-Yong, Elcio Abdalla, Phys. Lett. B 637, 357-361 (2006).

[32] N. Dalal, K. Abazajian, E. Jenkins, A. V. Manohar, Phys. Rev. Lett 86, 1939 (2001). 
[33] W. Zimdahl, D. Pavón, Gen. Rel. Grav. 35, 413-422 (2003).

[34] Amendola, L., Gannouji, R., Polarski, D., and Tsujikawa, S., Phys. Rev. D 75, 083504 (2007).

[35] Li, B., and Barrow, J.D., Phys. Rev. D 75, 084010 (2007).

[36] Hu, W., and Sawicki, I., Phys. Rev. D 76, 064004 (2007).

[37] Linder, E.V., Phys. Rev. D 80, 123528 (2009).

[38] Bean, R., 0909.3853 [astro-ph.CO], (2009).

[39] Tsujikawa, S., Uddin, K., and Tavakol, R., Phys. Rev. D 77, 043007 (2008).

[40] Zhang, P., Phys. Rev. D 73, 123504 (2006).

[41] Li, B., and Barrow, J.D., Phys. Rev. D 75, 084010 (2007).

[42] Tsujikawa, S., and Tatekawa, T., Phys. Lett. B 665, 325-331 (2008).

[43] Schmidt, F., Phys. Rev. D 78, 043002 (2008).

[44] J. H. He, B. Wang, E. Abdalla, 1109.1720 [gr-qc], (2011).

[45] M. S. Turner, F. Wilczek, Nature 298, 633-634 (1982).

[46] A. Kuzenko, P. Langacker, Phys. Lett. B 391, 29-33 (1997).

[47] W. D. Garretson, E. D. Carlson, Phys. Lett. B 315, 232-238 (1993).

[48] J. Wess, B. Zumino, Nucl. Phys. B 70, 39-50 (1974).

[49] L. D. Duffy, K. v. Bibber, New J. Phys. 11, 105008 (2009).

[50] L. Visinelli, P. Gondolo, Phys. Rev. D 80, 035024 (2009).

[51] P. Sikivie, 0909.0949 [hep-ph], (2009).

[52] L. Bergstrom, New J. Phys. 11, 105006 (2009).

[53] S. Coleman, Phys. Rev. D 15, 2929-2936 (1977). 
[54] D. J. Griffiths, "Introduction to Quantum Mechanics", Prentice Hall (1995).

[55] M. B. Paranjape, "Instanton Calculus", (2010) URL: http://users.aims.ac.za/ $\sim$ frederic/Quant_tun_instantons-1.pdf

[56] S. Coleman, Subnucl. Ser. 15, 805 (1979).

[57] C. G. Callan, S. Coleman, Phys. Rev. D 16, 1762-1768 (1977).

[58] S. Coleman, F. De Luccia, Phys. Rev. D 21, 3305-3315 (1980).

[59] A. Linde, Contemp. Concepts Phys. 5, 1-362 (2005) - seção 5.1. 